Research Article

\title{
Fractional K-BKZ Numerical Model of the Start-Up Flow for a Viscoelastic Shock Absorber
}

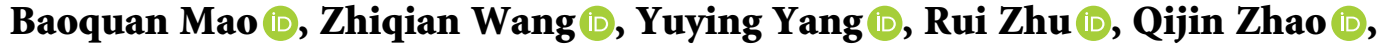 \\ and Bowen Zheng \\ Department of Weapons and Control Engineering, Army Academy of Armored Forces, Beijing 100072, China \\ Correspondence should be addressed to Zhiqian Wang; wangzhiqian1990@qq.com
}

Received 21 August 2019; Revised 13 January 2020; Accepted 18 January 2020; Published 22 February 2020

Academic Editor: Seungik Baek

Copyright $\odot 2020$ Baoquan Mao et al. This is an open access article distributed under the Creative Commons Attribution License, which permits unrestricted use, distribution, and reproduction in any medium, provided the original work is properly cited.

\begin{abstract}
This paper has proposed a fractional K-BKZ numerical model by adopting the framework of the classical K-BKZ model and the relaxation modulus of the fractional Maxwell model with quasiproperties to study the start-up flow of a viscoelastic shock absorber. The start-up flows in both the orifice and the gap of a shock absorber were simplified to unidirectional accelerated flows in a pipe and between two parallel plates where one plate is accelerating and the other is at rest. The fractional K-BKZ numerical model was then developed using the finite difference method with real-world initial and boundary conditions. Numerical simulation was then performed, and the results were validated through laboratory testing, based on a comparison of the maximum fluid level and the contact angle. The proposed fractional K-BKZ numerical model successfully simulated the characteristics of the viscoelastic material passing through the orifice or the gap of a shock absorber, as demonstrated by accurately capturing the change of the shape of the flow. This fractional K-BKZ numerical model provided better accuracy for the fluid's viscoelasticity and can be used for shock absorber design.
\end{abstract}

\section{Introduction}

Viscoelastic materials belong to the class of non-Newtonian pseudoplastic fluids. According to the mechanical structure of a shock absorber, the start-up flow forms of the viscoelastic material in the shock absorber can be divided into two types: the gap flow and the orifice flow. Due to the unique nonlinearity of viscoelastic fluid, many researchers have reviewed different research studies and viscoelastic materials and chose different mathematical models and constitutive relations to study the flow characteristics of viscoelastic fluids in a shock absorber. The research can be divided into two main categories: one involves empirical or semiempirical formula and the other involves classical models and their derived models.

Jia et al. $[1,2]$ used a linear fluid model and a power function to study the flow characteristics of a viscoelastic fluid between two parallel plates. The semiempirical formula suitable for the impact environment was derived experimentally, which can provide guidance for the damper design. Zhang et al. [3] combined the fractional Maxwell model with the semiempirical formula reported in the literature [1] to design a damper for heavy vehicles and validated the model's accuracy experimentally. Constantinou and Symans [4] studied a fluid damper that is used to make buildings earthquake-resistant and derived the empirical formula for experimental vibration environments. The model's results agreed well with the experimental results. However, due to the different mechanical structures and the operating environments of the damper and absorber in the above research, as well as the formula that was based on a large amount of experimental data, the derived empirical formulas had different forms as well as more restrictions in their use and limited scopes of application. Furthermore, the quantitative measurement for the start-up flow process of a viscoelastic fluid is difficult, so it is usually obtained through numerical simulation.

In the current research, the classical Maxwell model and its derivative models have attracted more and more attention. Especially with the introduction of the fractional derivative, the fractional Maxwell model has been validated and shown to have good applicability and can better simulate 
the flow process of a viscoelastic fluid $[5,6]$. Yang and Zhu [7] used the fractional Maxwell model to study the unidirectional start-up flow of a viscoelastic fluid from a state of rest and under a constant pressure gradient in an infinitely long pipe and obtained an exact solution. Furthermore, it has been proposed that when the fractional element exhibits solid-like behavior, the fluid that is simulated using the fractional Maxwell model also exhibits the characteristics of a solid. Yan et al. [8] adopted the fractional Maxwell method to study the oscillating flow in a pipe in rolling motion to simulate the conditions of a ship's pipelines subjected to seawater fluctuation; the velocity gradient and its variation of the oscillating flow of the pipe were then obtained. Tan et al. $[9,10]$ used the Laplace inverse transform with a continuous fractional derivative to discuss the fractional Maxwell model of a non-Newtonian viscoelastic fluid between two parallel plates when one plate undergoes random motion, and the analytical solutions for both the velocity and stress were then obtained. Carrera et al. [11] studied the fractional Maxwell model containing the relaxation process presenting non-Newtonian viscous behavior, and they analyzed and calculated the storage and loss modulus corresponding to the frequency and the amplitude function. Furthermore, they analyzed the stability of the function and found that the model's results agreed well with the experimental results. On the basis of the converse ladder Maxwell model, Yao [12] introduced the tensor and different fractional derivative definitions to apply the fractional dashpot model to the simulation for the flow process of a nonlinear viscoelastic fluid under large deformation. Through this method, the transient viscosity and modulus could be simulated by varying the parameters. The aforementioned studies can be divided into two categories: a velocity category and a modulus category. The former is used to obtain the relationship between velocity, time, stress, etc. Then the fluid's flow characteristics are characterized by the variation in velocity $[1,2,7-10]$; the latter is used to obtain the relationship between the modulus, viscosity, etc. as well as the frequency using the Fourier transform and other methods in order to characterize the rheological properties of the fluids $[11,12]$. The two categories are different since the former is more intuitive for the comprehension of the motion of a viscoelastic fluid, while the latter can better explain the rheological characteristics of a viscoelastic fluid through the perspective of its mechanical properties. However, when the fluid's velocity is higher, the fractional Maxwell model will gradually develop into a Newtonian fluid and lose viscoelasticity [13]. Similarly, when a viscoelastic fluid has a large deformation, the fractional Maxwell model is less nonlinear, which can lead to incorrect results [14]. A viscoelastic material belongs to the class of pseudoplastic fluids and the unique working characteristics of a shock absorber causes the viscoelastic fluid to have a higher velocity, so there are certain limitations to the fractional Maxwell model when simulating the flow characteristics of a viscoelastic material in a shock absorber.

The K-BKZ constitutive equation is a relatively new model [15]. Many researchers have found that the K-BKZ model can describe nonlinear viscoelastic behavior very well [16-19]. Some researchers [20, 21] modified the K-BKZ model and derived the Wagner integral constitutive equation by ignoring the Cauchy tensor in the constitutive equation. The accuracy and consistency of the Wagner integral model prediction were demonstrated experimentally. Yamaguchi [22] used both the Oldroyd-B model and the K-BKZ model to simulate the flow conditions of an incompressible viscoelastic fluid within the inlet region of a circular tube. Bower et al. [23] used the K-BKZ model to simulate a fluid's shear thinning effect for a specific fluid type, which can provide substantial insights about the combined effect of inertia and shear thinning in an orthogonal rheometer and provide predictive guidance for similar fluid models. Ebrahimi [24] et al. used the K-BKZ constitutive models to predict the transient shear response of high-density polyethylene melt. The comparison between the simulated results and the experimental data, obtained by a rotational rheometer, showed good agreement. By using the finite difference method, Tomé [25] et al. obtained the numerical solution to the K-BKZ integral constitutive equation with $2 \mathrm{D}$ free-surface motion, and the convergence of the mesh refinement in the numerical solution was validated and evaluated. The K-BKZ model with the relaxation kernel is described by power law function has the accuracy of the viscoelasticity model only at short times [14] and [26]. Jaishankar and McKinley [14] proposed a fractional K-BKZ model, which provided an accurate method to complete the transformation between the linear and nonlinear rheological study of multiscale materials. The model's accuracy has been validated experimentally, which proved that the fractional K-BKZ model can effectively capture the shear thinning phenomenon of a viscoelastic fluid and maintain the good nonlinearity of the viscoelastic fluid.

A viscoelastic material belongs to the class of pseudoplastic fluids and its flow inside a shock absorber has a larger flow velocity and a strong viscoelasticity, which can be studied using a K-BKZ model, especially the fractional $\mathrm{K}-\mathrm{BKZ}$ model. Most of the related studies were focused on obtaining the analytical solutions of the K-BKZ model, except for two studies [13, 25], where numerical solutions were derived to obtain the models. However, the solution of the fractional K-BKZ model involves fractional calculus equations, and the process for solving the analytical solution is difficult and more complicated, so computational simulation is not easy. Comparatively, simulation on the basis of a numerical solution is easy to implement. The use of the fractional K-BKZ model to simulate the unidirectional accelerated start-up flows of viscoelastic fluid in a pipe has rarely been reported. Meanwhile, in the engineering field, it has been rarely reported that the fractional K-BKZ model was applied to a study on the start-up flow of a shock absorber.

Therefore, aimed at the start-up flow of a viscoelastic material inside the orifice and gap of a shock absorber, the present paper has simplified the flow processes in both the orifice and the gap to accelerated motion in a pipe and the flow between a moving plate and a static plate. Subsequently, 
the fractional K-BKZ numerical model was constructed by adopting the framework of the classical K-BKZ model and the relaxation modulus of the fractional Maxwell model with quasiproperties. Comparative analysis of classical K-BKZ numerical model, the fractional Maxwell numerical model, and the Newtonian fluid model have been performed, and the unidirectional accelerated start-up flows of viscoelastic fluid in the orifice and the gap have been studied. A start-up flow test system was designed, and it captured the flow process of a viscoelastic material in the orifice of a shock absorber when the flow starts up, which validated the accuracy of the fractional K-BKZ numerical model.

\section{Development of the Fractional K-BKZ Start- Up Flow Numerical Model}

2.1. The Fractional K-BKZ Model. The classical K-BKZ constitutive equation is expressed as [26, 27]

$$
\sigma(t)=\int_{-\infty}^{t}\left[2 \frac{\partial W\left(t-s, I_{1}, I_{2}\right)}{\partial I_{1}} C^{-1}(t, s)-2 \frac{\partial W\left(t-s, I_{1}, I_{2}\right)}{\partial I_{2}} C(t, s)\right] \mathrm{d} s,
$$

where $C^{-1}=\left(F^{-1}\right)^{T}$ is the relative Finger strain tensor [26]. $C$ is the relative Cauchy strain tensor. $I_{1}$ and $I_{2}$ are the first and second invariants, respectively, of $C^{-1} . W\left(t-s, I_{1}, I_{2}\right)$ is the potential function related to the strain energy function of the material can be factored into a time-dependent memory function $m(t-s)$ and a scalar potential $w\left(I_{1}, I_{2}\right)$, i.e., $W(t-$ $\left.s, I_{1}, I_{2}\right)=m(t-s) w\left(I_{1}, I_{2}\right)$ [20, 28]. Wagner proposed $[20,26-28]$ that the second normal shear stress difference is not considered; that is, the relative Cauchy strain tensor is ignored, i.e., $\left(\left(\partial w\left(I_{1}, I_{2}\right)\right) / \partial I_{2}\right)=0[20,28]$, the Wagner model can be written as [20, 26-28]:

$$
\sigma(t)=\int_{-\infty}^{t} m(t-s) h\left(I_{1}, I_{2}\right) C^{-1}(t, s) \mathrm{d} s,
$$

where $h\left(I_{1}, I_{2}\right)=2\left(\left(\partial w\left(I_{1}, I_{2}\right)\right) / \partial I_{1}\right)[20,28]$. In the unidirectional flow, the relative Finger strain tensor can be written as $[27,29,30]$

$$
C^{-1}=\left(\begin{array}{ccc}
1+\varepsilon^{2}(t, s) & \varepsilon(t, s) & 0 \\
\varepsilon(t, s) & 1 & 0 \\
0 & 0 & 1
\end{array}\right),
$$

then the expression of the shear stress can be simplified to $[14,20,21,27,30]$

$$
\sigma(t)=\int_{-\infty}^{t} m(t-s) h(\varepsilon) \varepsilon(t, s) \mathrm{d} s,
$$

where $m(t-s)$ is related to the relaxation modulus $G(t-s)$ and can be written as $[14,28]$

$$
m(t-s)=\frac{\mathrm{d} G(t-s)}{\mathrm{d} s} .
$$

Using the relaxation modulus of the fractional Maxwell constitutive equation gives [14]

$$
G(t)=\psi t^{-\beta} E_{\alpha-\beta, 1-\beta}\left(-\frac{\psi}{\kappa} t^{\alpha-\beta}\right),
$$

where $(\kappa, \alpha)$ and $(\psi, \beta)$ denote two mechanical elements of the spring-pots, $\alpha$ and $\beta$ are fractional orders, and $0 \leq \beta<\alpha \leq 1 ; \kappa$ and $\psi$ are quasiproperties, and their units are $\mathrm{Pa} \cdot \mathrm{s}^{\alpha}$ and $\mathrm{Pa} \cdot \mathrm{s}^{\beta}$, respectively; $E_{b, c}(z)$ is the generalized Mittag-Leffler function, and $b=\alpha-\beta>0, c=1-\beta>0$, $z \in C$, which is defined as $[31,32]$

$$
E_{b, c}(z)=\sum_{i=0}^{\infty} \frac{z^{i}}{\Gamma(b i+c)} .
$$

Through simultaneous solutions (6) and (7), the memory function (5) can be written as [14]

$$
m(t-s)=-\psi(t-s)^{-1-\beta} E_{\alpha-\beta,-\beta}\left(-\frac{\psi}{\kappa}(t-s)^{\alpha-\beta}\right),
$$

and here the form of the damping function is [28]

$$
h(\varepsilon)=\frac{1}{1+a \varepsilon^{2}(t, s)},
$$

where $\varepsilon(t, s)$ is the relative shear strain between any two moments, which can be defined as [14]

$$
\varepsilon(t, s)=\varepsilon(t)-\varepsilon(s)=\dot{\varepsilon}(t-s) .
$$

Substituting (8) and (9) into (4), the fractional K-BKZ model can be written as [14]

$$
\begin{aligned}
\sigma(t)= & -\int_{-\infty}^{t} \psi(t-s)^{-1-\beta} E_{\alpha-\beta,-\beta}\left(-\frac{\psi}{\kappa}(t-s)^{\alpha-\beta}\right) \\
& \cdot \frac{\varepsilon(t, s)}{1+a \varepsilon^{2}(t, s)} \mathrm{d} s .
\end{aligned}
$$

2.2. Structure and Simplification. The geometrical schematic of a viscoelastic shock absorber has been shown in Figure 1 . The shock absorber was installed as a stressed structure by the shock absorber's stand (Figure 1(h)). The viscoelastic material was injected into the shock absorber's chamber (Figure 1(b)) through the viscoelastic material inlet (Figure 1(a)). The volume of the viscoelastic material filling was adjusted as needed to give the shock absorber a certain prestress, which was called the initial preload force. When the external impact force was greater than the initial preload force, the piston rod (Figure 1(e)) was pushed and drove the piston (Figure 1(d)) to compress the viscoelastic material in the shock absorber's chamber (Figure 1(b)). The viscoelastic material flowed through the gap (Figure 1(c)) and the orifice (Figure 1(f)) of the piston after compression that generated the viscous damping force which hindered the piston from moving forward, and this process converted part of the external force into heat energy which was lost and potential energy which was stored. When the external force was withdrawn, that is, when the impact force was less than the initial preload force, the viscoelastic material expanded by 


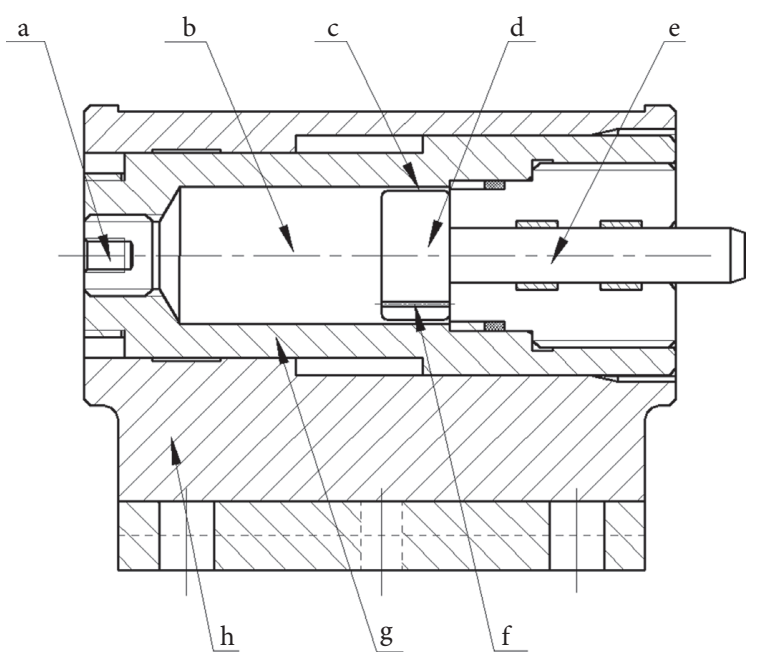

Figure 1: The geometrical schematic of a viscoelastic shock absorber. (a) The viscoelastic material inlet. (b) The shock absorber's chamber. (c) The gap. (d) The piston. (e) The piston rod. (f) The orifice. (g) The shock absorber's chamber wall. (h) The shock absorber's stand.

itself and released the potential energy stored from the compression and some part of the potential energy converted to heat energy, while the rest potential energy pushed the piston back to its original position. The viscoelastic material flowed back to its initial state before compression through the orifice and the gap, and was ready for the next impact.

In order to meet the actual working conditions of the start-up flow of a viscoelastic material in a shock absorber, the flow relations of the start-up flow of a viscoelastic material in the orifice and gap can be simplified into the two-dimensional space. The former (Figure 1(f)) can be considered as the accelerated flow of a fluid in a pipe (see Figure 2(a)) while the latter (Figure 1(c)) can be considered as the flow of a fluid between two parallel plates while one plate is at rest and the other is accelerating (see Figure 2(b)).

2.3. Governing Equation. The time rate of change of the total momentum in the fluid's motion is equal to the addition of its total volume force and its interactive surface force. The acceleration can be considered as the time rate of the momentum of the fluid having unit mass. According to Newton's law of motion and the momentum balance theorem, the following can be obtained:

$$
\mathrm{MA}=\sum F
$$

The vector expression of the motion equation that is irrelevant to the coordinate system can be expressed as [33]

$$
\rho \frac{\partial}{\partial t} U=\rho f+\nabla \cdot \sigma
$$

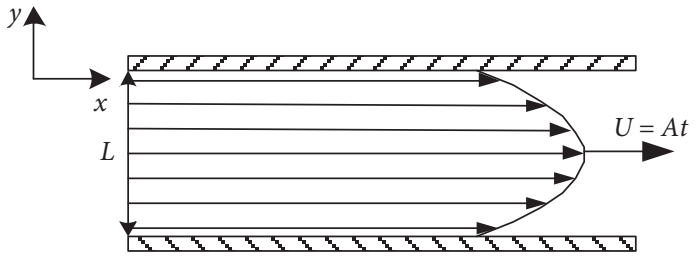

(a)

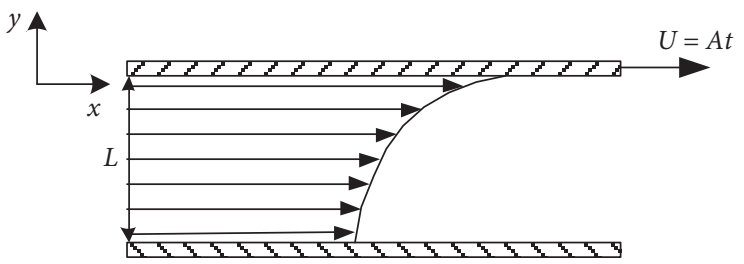

(b)

FIGURE 2: Simplifying the relationship of the motion between a shock absorber's structure and the viscoelastic material. (a) The motion relationship between an orifice and the viscoelastic fluid and (b) a gap and the viscoelastic fluid.

where $U$ is the flow velocity, $f$ represents the unit volume force, $\rho$ is the density, and $\sigma$ is the stress tensor.

Thus, the volume force is negligible and can be ignored. Then, the following can be obtained:

$$
\rho \frac{\partial}{\partial t} U=\nabla \cdot \sigma
$$

The velocity is only distributed in the $y$ direction (see Figure 2), so

$$
\rho \frac{\partial u(t, y)}{\partial t}=\frac{\partial \sigma(t, y)}{\partial y}
$$

When $t \leq 0, \varepsilon(t)=0,(11)$ can be written as

$$
\begin{aligned}
\sigma(t)= & -\psi \int_{0}^{t} \theta^{-1-\beta} E_{\alpha-\beta, 1-\beta}\left(-\frac{\psi}{\kappa} \theta^{\alpha-\beta}\right) \\
& \cdot \frac{\varepsilon(t)-\varepsilon(t-\theta)}{1+a(\varepsilon(t-\theta)-\varepsilon(t))^{2}} \mathrm{~d} \theta \\
& +\psi \int_{t}^{\infty} \theta^{-1-\beta} E_{\alpha-\beta, 1-\beta}\left(-\frac{\psi}{\kappa} \theta^{\alpha-\beta}\right) \frac{\varepsilon(t)}{1+a \varepsilon^{2}(t)} \mathrm{d} \theta
\end{aligned}
$$

Since the definition of the velocity gradient is $(\mathrm{d} u / \mathrm{d} y)=\dot{\varepsilon}$, the strain definition is $\varepsilon(t, y)=\int_{0}^{t}((\partial \varepsilon(s, y)) / \partial s) \mathrm{d} s$, and the definition of the Mittag-Leffler function derivatives is $\quad(\mathrm{d} / \mathrm{d} s)\left(\theta^{-\beta} E_{\alpha-\beta, 1-\beta}\left(-\left((\kappa / \psi) \theta^{\alpha-\beta}\right)\right)\right)=\theta^{-1-\beta} E_{\alpha-\beta,-\beta}$ $\left(-\left((\kappa / \psi) \theta^{\alpha-\beta}\right)\right) \mathrm{d} s$ [32]; the fractional K-BKZ governing equation can be written as 


$$
\begin{aligned}
\sigma(t, y)= & -\psi \int_{0}^{t} \theta^{-1-\beta} E_{\alpha-\beta,-\beta}\left(-\frac{\psi}{\kappa} \theta^{\alpha-\beta}\right) \frac{\int_{t-\theta}^{t}((\partial u(w, y)) / \partial y) \mathrm{d} w}{1+a\left(\int_{t-\theta}^{t}((\partial u(w, y)) / \partial y) \mathrm{d} w\right)^{2}} \mathrm{~d} \theta \\
& +\psi t^{-\beta} E_{\alpha-\beta, 1-\beta}\left(-\frac{\psi}{\kappa} t^{\alpha-\beta}\right) \frac{\int_{0}^{t}((\partial u(w, y)) / \partial y) \mathrm{d} w}{1+a\left(\int_{0}^{t}((\partial u(w, y)) / \partial y) \mathrm{d} w\right)^{2}}, \\
\frac{\partial \sigma(t, y)}{\partial y}= & \rho \frac{\partial u(t, y)}{\partial t} \\
= & -\psi \int_{0}^{t} \theta^{-1-\beta} E_{\alpha-\beta,-\beta}\left(-\frac{\psi}{\kappa} \theta^{\alpha-\beta}\right) \frac{\int_{t-s}^{t}\left(\left(\partial^{2} u(w, y)\right) / \partial y^{2}\right) \mathrm{d} w}{1+a\left(\int_{t-s}^{t}((\partial u(w, y)) / \partial y) \mathrm{d} w\right)^{2}}\left\{\begin{array}{c}
2 a\left(\int_{t t-s}^{t}((\partial u(w, y)) / \partial y) \mathrm{d} w\right)^{2} \\
1+a\left(\int_{t-s}^{t}((\partial u(w, y)) / \partial y) \mathrm{d} w\right)^{2}
\end{array}\right\} \mathrm{ds} \\
& +\psi t^{-\beta} E_{\alpha-\beta, 1-\beta}\left(-\frac{\psi}{\kappa} t^{\alpha-\beta}\right) \frac{\int_{0}^{t}\left(\left(\partial^{2} u(w, y)\right) / \partial y^{2}\right) \mathrm{d} w}{1+a\left(\int_{0}^{t}((\partial u(w, y)) / \partial y) \mathrm{d} w\right)^{2}}\left\{1-\frac{2 a\left(\int_{0}^{t}((\partial u(w, y)) / \partial y) \mathrm{d} w\right)^{2}}{1+a\left(\int_{0}^{t}((\partial u(w, y)) / \partial y) \mathrm{d} w\right)^{2}}\right\} .
\end{aligned}
$$

2.4. The Initial Conditions and the Boundary Conditions. When $t \leq 0, u(t)=0$. In the orifice, the flow velocity of the start-up flow at the center gradually increases with acceleration $A$, while the velocity near the pipe wall is always zero. In the gap, the flow velocity near the side of the moving plate increases with acceleration $A$, while the velocity near the stationary plate is always zero. So the initial conditions and the boundary conditions are as follows:

(1) The orifice:

$$
\begin{cases}u(t, 0)=0, & t>0 \\ u(t, L)=0, & t>0 \\ u\left(t, \frac{L}{2}\right)=A t, & t>0 \\ u(0, y)=0, & y>0 \\ \frac{\partial u(0, y)}{\partial t}=0, & y>0\end{cases}
$$

(2) The gap:

$$
\begin{cases}u(t, 0)=A t, & t>0, \\ u(t, L)=0, & t>0, \\ u(0, y)=0, & y>0, \\ \frac{\partial u(0, y)}{\partial t}=0, & y>0\end{cases}
$$

where $L$ is the distance of the gap and the orifice and $A$ is the acceleration.

2.5. Dimensionless Analysis. According to the $\Pi$ theorem, the dimensionless variables of the fractional $\mathrm{K}-\mathrm{BKZ}$ model can be obtained as

$$
\begin{aligned}
u^{*} & =\left(\frac{\psi}{\kappa}\right)^{1 /(\alpha-\beta)} \frac{u}{A}, \\
y^{*} & =L^{-1} y, \\
t^{*} & =\left(\frac{\psi}{\kappa}\right)^{1 /(\alpha-\beta)} t, \\
\sigma^{*} & =\frac{\sigma}{L \rho A}, \\
a^{*} & =a\left(\frac{A}{L}\right)^{2}\left(\frac{\kappa}{\psi}\right)^{4 /(\alpha-\beta)}, \\
\vartheta & =\frac{\psi}{L^{2} \rho}\left(\frac{\kappa}{\psi}\right)^{(2-\beta) /(\alpha-\beta)} .
\end{aligned} .
$$

Substituting the dimensionless variables in (21) in (17) and (18), the fractional K-BKZ start-up flow dimensionless equation can be obtained (for brevity, the dimensionless mark “*” is omitted): 


$$
\begin{aligned}
\sigma(t, y)= & -\vartheta \int_{0}^{t} \theta^{-1-\beta} E_{\alpha-\beta,-\beta}\left(-\theta^{\alpha-\beta}\right) \frac{\int_{t-\theta}^{t}((\partial u(w, y)) / \partial y) \mathrm{d} w}{1+a\left(\int_{t-\theta}^{t}((\partial u(w, y)) / \partial y) \mathrm{d} w\right)^{2}} \mathrm{~d} \theta \\
& +9 t^{-\beta} E_{\alpha-\beta, 1-\beta}\left(-t^{\alpha-\beta}\right) \frac{\int_{0}^{t}((\partial u(w, y)) / \partial y) \mathrm{d} w}{1+a\left(\int_{0}^{t}((\partial u(w, y)) / \partial y) \mathrm{d} w\right)^{2}} \\
\frac{\partial u(t, y)}{\partial t}= & -\vartheta \int_{0}^{t} \theta^{-1-\beta} E_{\alpha-\beta,-\beta}\left(-\theta^{\alpha-\beta}\right) \frac{\int_{t-s}^{t}\left(\left(\partial^{2} u(w, y)\right) / \partial y^{2}\right) \mathrm{d} w}{1+a\left(\int_{t-s}^{t}((\partial u(w, y)) / \partial y) \mathrm{d} w\right)^{2}}\left\{\begin{array}{c}
2 a\left(\int_{t-s}^{t}((\partial u(w, y)) / \partial y) \mathrm{d} w\right)^{2} \\
1+a\left(\int_{t-s}^{t}((\partial u(w, y)) / \partial y) \mathrm{d} w\right)^{2}
\end{array}\right\} \mathrm{d} s \\
& +9 t^{-\beta} E_{\alpha-\beta, 1-\beta}\left(-t^{\alpha-\beta}\right) \frac{\int_{0}^{t}\left(\left(\partial^{2} u(w, y)\right) / \partial y^{2}\right) \mathrm{d} w}{1+a\left(\int_{0}^{t}((\partial u(w, y)) / \partial y) \mathrm{d} w\right)^{2}}\left\{1-\frac{2 a\left(\int_{0}^{t}((\partial u(w, y)) / \partial y) \mathrm{d} w\right)^{2}}{1+a\left(\int_{0}^{t}((\partial u(w, y)) / \partial y) \mathrm{d} w\right)^{2}}\right\}
\end{aligned}
$$

The corresponding dimensionless initial conditions and boundary conditions of the orifice and gap are given as

$$
\begin{aligned}
& \begin{cases}u(t, 0)=0, & t>0, \\
u(t, 1)=0, & t>0, \\
u\left(t, \frac{1}{2}\right)=t, & t>0, \\
u(0, y)=0, & y>0, \\
\frac{\partial u(0, y)}{\partial t}=0, & y>0,\end{cases} \\
& \begin{cases}u(t, 0)=t, & t>0, \\
u(t, 1)=0, & t>0, \\
u(0, y)=0, & y>0, \\
\frac{\partial u(0, y)}{\partial t}=0, & y>0 .\end{cases}
\end{aligned}
$$

Let $u_{j}^{i}$ and $\sigma_{j}^{i}$ denote $u\left(t_{i}, y_{j}\right)$ and $\sigma\left(t_{i}, y_{j}\right)$. As $F\left(t_{k}\right)=$ $t_{k}^{-1-\beta} E_{\alpha-\beta,-\beta}\left(-t_{k}^{\alpha-\beta}\right)$ and $H\left(t_{k}\right)=t_{k}^{-\beta} E_{\alpha-\beta, 1-\beta}\left(-t_{k}^{\alpha-\beta}\right)$, the fractional K-BKZ start-up flow numerical model can be written as

$$
\begin{aligned}
\sigma_{n}^{m}=-\vartheta \sum_{i=1}^{m}\left\{F\left(t_{i}\right) \frac{\left((\Delta t)^{2} / \Delta y\right) \sum_{j=m-i+1}^{m}\left(u_{n+1}^{j}-u_{n}^{j}\right)}{1+a(\Delta t / \Delta y)^{2}\left(\sum_{j=m-i+1}^{m}\left(u_{n+1}^{j}-u_{n}^{j}\right)\right)^{2}}\right\}+\vartheta H\left(t_{m}\right) \frac{(\Delta t / \Delta y) \sum_{j=1}^{m}\left(u_{n+1}^{j}-u_{n}^{j}\right)}{1+a(\Delta t / \Delta y)^{2}\left(\sum_{j=1}^{m}\left(u_{n+1}^{j}-u_{n}^{j}\right)\right)^{2}}, \\
\frac{u_{m+1, n}-u_{m, n}}{\Delta t}=-\vartheta \sum_{i=1}^{m} F\left(t_{i}\right) \frac{(\Delta t / \Delta y)^{2} \sum_{j=m-i+1}^{m}\left(u_{j, n+1}-2 u_{j, n}+u_{j, n-1}\right)}{1+a(\Delta t / \Delta y)^{2}\left(\sum_{j=m-i+1}^{m}\left(u_{j, n+1}-u_{j, n}\right)\right)^{2}}\left\{1-\frac{2 a(\Delta t / \Delta y)^{2}\left(\sum_{j=m-i+1}^{m}\left(u_{j, n+1}-u_{j, n}\right)\right)^{2}}{1+a(\Delta t / \Delta y)^{2}\left(\sum_{j=m-i+1}^{m}\left(u_{j, n+1}-u_{j, n}\right)\right)^{2}}\right\} \\
+\vartheta H\left(t_{m}\right) \frac{\left(\Delta t /(\Delta y)^{2}\right) \sum_{j=1}^{m}\left(u_{j, n+1}-2 u_{j, n}+u_{j, n-1}\right)}{1+a(\Delta t / \Delta y)^{2}\left(\sum_{j=1}^{m}\left(u_{j, n+1}-u_{j, n}\right)\right)^{2}}\left\{1-\frac{2 a(\Delta t / \Delta y)^{2}\left(\sum_{j=1}^{m}\left(u_{j, n+1}-u_{j, n}\right)\right)^{2}}{1+a(\Delta t / \Delta y)^{2}\left(\sum_{j=1}^{m}\left(u_{j, n+1}-u_{j, n}\right)\right)^{2}}\right\} .
\end{aligned}
$$




\section{Development of the Classical K-BKZ Start-Up Flow Numerical Model}

Larson expressed the relaxation modulus describing polymers in the K-BKZ model as [34]

$$
G(t)=c t^{-g},
$$

where $c$ and $g$ are constants obtained through experiments, respectively.

The determination of the values of $c$ and $g$ is usually based on a grate quantity of experimental data. Therefore, it would be difficult to use equation (28) for accurate numerical calculation in this research due to the lack of experimental data.

Consequently, Jaishankar et al. [14, 30, 35] introduced the fractional element model into the K-BKZ model and used fractional power law function to represent relaxation modulus as follows:

$$
G(t)=\frac{\psi t^{-\beta}}{\Gamma(1-\beta)},
$$

The memory function (5) can be written as

$$
m(t-s)=\frac{\psi \beta}{\Gamma(1-\beta)}(t-s)^{-1-\beta} .
$$

Thus, the K-BKZ model can be written as

$$
\sigma(t)=\int_{-\infty}^{t} \frac{\psi \beta}{\Gamma(1-\beta)}(t-s)^{-1-\beta} \frac{\varepsilon(t, s)}{1+a \varepsilon^{2}(t, s)} \mathrm{d} s .
$$

Using the methods in Section 2, the classical K-BKZ governing equation can be written as

$$
\begin{aligned}
\sigma(t, y)= & \frac{\psi \beta}{\Gamma(1-\beta)} \int_{0}^{t} \theta^{-1-\beta} \frac{\int_{t-\theta}^{t}((\partial u(w, y)) / \partial y) \mathrm{d} w}{1+a\left(\int_{t-\theta}^{t}((\partial u(w, y)) / \partial y) \mathrm{d} w\right)^{2}} \mathrm{~d} \theta+\frac{\psi}{\Gamma(1-\beta)} t^{-\beta} \frac{\int_{0}^{t}((\partial u(w, y)) / \partial y) \mathrm{d} w}{1+a\left(\int_{0}^{t}((\partial u(w, y)) / \partial y) \mathrm{d} w\right)^{2}}, \\
\frac{\partial \sigma(t, y)}{\partial y}= & \rho \frac{\partial u(t, y)}{\partial t} \\
= & \frac{\psi \beta}{\Gamma(1-\beta)} \int_{0}^{t} \theta^{-1-\beta} \frac{\int_{t-s}^{t}\left(\left(\partial^{2} u(w, y)\right) / \partial y^{2}\right) \mathrm{d} w}{1+a\left(\int_{t-s}^{t}((\partial u(w, y)) / \partial y) \mathrm{d} w\right)^{2}}\left\{1-\frac{2 a\left(\int_{t-s}^{t}((\partial u(w, y)) / \partial y) \mathrm{d} w\right)^{2}}{1+a\left(\int_{t-s}^{t}((\partial u(w, y)) / \partial y) \mathrm{d} w\right)^{2}}\right\} \mathrm{d} s \\
& +\frac{\psi}{\Gamma(1-\beta)} t^{-\beta} \frac{\int_{0}^{t}\left(\left(\partial^{2} u(w, y)\right) / \partial y^{2}\right) \mathrm{d} w}{1+a\left(\int_{0}^{t}((\partial u(w, y)) / \partial y) \mathrm{d} w\right)^{2}}\left\{1-\frac{2 a\left(\int_{0}^{t}((\partial u(w, y)) / \partial y) \mathrm{d} w\right)^{2}}{1+a\left(\int_{0}^{t}((\partial u(w, y)) / \partial y) \mathrm{d} w\right)^{2}}\right\} .
\end{aligned}
$$

and the classical K-BKZ start-up flow dimensionless equation can be obtained (for brevity, the dimensionless mark “*” is omitted):

$$
\begin{aligned}
\sigma(t, y)= & \frac{\vartheta \beta}{\Gamma(1-\beta)} \int_{0}^{t} \theta^{-1-\beta} \frac{\int_{t-\theta}^{t}((\partial u(w, y)) / \partial y) \mathrm{d} w}{1+a\left(\int_{t-\theta}^{t}((\partial u(w, y)) / \partial y) \mathrm{d} w\right)^{2}} \mathrm{~d} \theta+\frac{\vartheta}{\Gamma(1-\beta)} t^{-\beta} \frac{\int_{0}^{t}((\partial u(w, y)) / \partial y) \mathrm{d} w}{1+a\left(\int_{0}^{t}((\partial u(w, y)) / \partial y) \mathrm{d} w\right)^{2}}, \\
\frac{\partial u(t, y)}{\partial t}= & \vartheta \int_{0}^{t} \theta^{-1-\beta} E_{\alpha-\beta,-\beta}\left(-\theta^{\alpha-\beta}\right) \frac{\int_{t-s}^{t}\left(\left(\partial^{2} u(w, y)\right) / \partial y^{2}\right) \mathrm{d} w}{1+a\left(\int_{t-s}^{t}((\partial u(w, y)) / \partial y) \mathrm{d} w\right)^{2}}\left\{1-\frac{2 a\left(\int_{t-s}^{t}((\partial u(w, y)) / \partial y) \mathrm{d} w\right)^{2}}{1+a\left(\int_{t-s}^{t}((\partial u(w, y)) / \partial y) \mathrm{d} w\right)^{2}}\right\} d s \\
& +9 t^{-\beta} \frac{\int_{0}^{t}\left(\left(\partial^{2} u(w, y)\right) / \partial y^{2}\right) \mathrm{d} w}{1+a\left(\int_{0}^{t}((\partial u(w, y)) / \partial y) \mathrm{d} w\right)^{2}}\left\{1-\frac{2 a\left(\int_{0}^{t}((\partial u(w, y)) / \partial y) \mathrm{d} w\right)^{2}}{1+a\left(\int_{0}^{t}((\partial u(w, y)) / \partial y) \mathrm{d} w\right)^{2}}\right\} .
\end{aligned}
$$


Then the classical K-BKZ start-up flow numerical model can be written as

$$
\begin{aligned}
\sigma_{n}^{m}= & \frac{\vartheta \beta}{\Gamma(1-\beta)} \sum_{i=1}^{m}\left\{\frac{t_{i}^{-1-\beta}\left((\Delta t)^{2} / \Delta y\right) \sum_{j=m-i+1}^{m}\left(u_{n+1}^{j}-u_{n}^{j}\right)}{1+a(\Delta t / \Delta y)^{2}\left(\sum_{j=m-i+1}^{m}\left(u_{n+1}^{j}-u_{n}^{j}\right)\right)^{2}}\right\}+\frac{9 t_{m}^{-\beta}}{\Gamma(1-\beta)} \frac{(\Delta t / \Delta y) \sum_{j=1}^{m}\left(u_{n+1}^{j}-u_{n}^{j}\right)}{1+a(\Delta t / \Delta y)^{2}\left(\sum_{j=1}^{m}\left(u_{n+1}^{j}-u_{n}^{j}\right)\right)^{2}}, \\
\frac{u_{m+1, n}-u_{m, n}}{\Delta t}= & \frac{\vartheta \beta}{\Gamma(1-\beta)} \sum_{i=1}^{m} \frac{t_{i}^{-1-\beta}(\Delta t / \Delta y)^{2} \sum_{j=m-i+1}^{m}\left(u_{j, n+1}-2 u_{j, n}+u_{j, n-1}\right)}{1+a(\Delta t / \Delta y)^{2}\left(\sum_{j=m-i+1}^{m}\left(u_{j, n+1}-u_{j, n}\right)\right)^{2}}\left\{1-\frac{2 a(\Delta t / \Delta y)^{2}\left(\sum_{j=m-i+1}^{m}\left(u_{j, n+1}-u_{j, n}\right)\right)^{2}}{1+a(\Delta t / \Delta y)^{2}\left(\sum_{j=m-i+1}^{m}\left(u_{j, n+1}-u_{j, n}\right)\right)^{2}}\right\} \\
& +\frac{\vartheta t_{m}^{-\beta}}{\Gamma(1-\beta)} \frac{\left(\Delta t /(\Delta y)^{2}\right) \sum_{j=1}^{m}\left(u_{j, n+1}-2 u_{j, n}+u_{j, n-1}\right)}{1+a(\Delta t / \Delta y)^{2}\left(\sum_{j=1}^{m}\left(u_{j, n+1}-u_{j, n}\right)\right)^{2}}\left\{1-\frac{2 a(\Delta t / \Delta y)^{2}\left(\sum_{j=1}^{m}\left(u_{j, n+1}-u_{j, n}\right)\right)^{2}}{1+a(\Delta t / \Delta y)^{2}\left(\sum_{j=1}^{m}\left(u_{j, n+1}-u_{j, n}\right)\right)^{2}}\right\} .
\end{aligned}
$$

The corresponding dimensionless initial conditions and boundary conditions of the orifice and gap are given as (24) and (25).

\section{Development of the Fractional Maxwell Start- Up Flow Numerical Model}

The fractional Maxwell model is proposed in $[18,30,36,37]$ :

$$
\sigma+\frac{\kappa}{\psi} D_{t}^{\alpha-\beta} \sigma=\kappa D_{t}^{\alpha} \varepsilon
$$

According to the methods in Section 2, the fractional Maxwell governing equation can be written as

$$
\begin{array}{r}
\sigma(t, y)+\frac{\kappa}{\psi} D_{t}^{\alpha-\beta} \sigma(t, y)=\kappa J_{t}^{1-\alpha}\left(\frac{\partial u(t, y)}{\partial y}\right), \\
\rho \frac{\partial u(t, y)}{\partial t}+\rho \frac{\kappa}{\psi} D_{t}^{\alpha-\beta+1} u(t, y)=\kappa J_{t}^{1-\alpha}\left(\frac{\partial^{2} u(t, y)}{\partial^{2} y}\right) .
\end{array}
$$

and the fractional Maxwell start-up flow dimensionless equation can be obtained (for brevity, the dimensionless mark “*” is omitted):

$$
\sigma+D_{t}^{\alpha-\beta} \sigma(t, y)=\vartheta J_{t}^{1-\alpha} \frac{\partial u(t, y)}{\partial y},
$$

$$
\frac{\partial u(t, y)}{\partial t}+D_{t}^{\alpha-\beta+1} u(t, y)=\vartheta J_{t}^{1-\alpha}\left(\frac{\partial^{2} u(t, y)}{\partial^{2} y}\right)
$$

Applying the finite difference method in the literature [38] to discretize and approximate (37) and (38), the fractional Maxwell start-up flow numerical model can be written as

$$
\begin{gathered}
\sigma_{n}^{k+1}+\frac{(\Delta t)^{\beta-\alpha}}{\Gamma(2-\alpha+\beta)}\left\{\sigma_{n}^{k+1}+\sum_{j=1}^{k}\left[(j+1)^{1-\alpha+\beta}-2 j^{1-\alpha+\beta}+(j-1)^{1-\alpha+\beta}\right] \sigma_{n}^{k-j+1}\right\} \\
=\vartheta \frac{(\Delta t)^{1-\alpha}}{\Gamma(2-\alpha) \cdot \Delta y} \sum_{j=0}^{k}\left[(k-j+1)^{1-\alpha}-(k-j)^{1-\alpha}\right]\left(u_{n+1}^{j}-u_{n}^{j}\right), \\
\frac{u_{n}^{k+1}-u_{n}^{k}+\frac{(\Delta t)^{\beta-\alpha-1}}{\Gamma t}\left\{u_{n}^{k+1}-u_{n}^{k}+\sum_{j=1}^{k}\left[(j+1)^{1-\alpha+\beta}-2 j^{1-\alpha+\beta}+(j-1)^{1-\alpha+\beta}\right]\left[u_{n}^{k-j+1}-u_{n}^{k-j}\right]\right\}}{=\vartheta \frac{(\Delta t)^{1-\alpha}}{\Gamma(2-\alpha) \cdot(\Delta y)^{2}} \sum_{j=0}^{k}\left[(k-j+1)^{1-\alpha}-(k-j)^{1-\alpha}\right]\left(u_{n+1}^{j}+u_{n-1}^{j}-2 u_{n}^{j}\right),}
\end{gathered}
$$

where (39) and (40) should satisfy $0<\beta<\alpha<1$. The corresponding dimensionless initial conditions and boundary conditions of the orifice and gap are given as (24) and (25).
When there is only one spring-pot element and $\alpha=1$, then the difference approximation to the (37) and (38) can be written as 


$$
\begin{aligned}
\sigma_{n}^{m+1} & =\frac{\vartheta}{\Delta y}\left(u_{n+1}^{m}-u_{n}^{m}\right), \\
\frac{u_{n}^{m+1}-u_{n}^{m}}{\Delta t} & =\frac{\vartheta}{(\Delta y)^{2}}\left(u_{n+1}^{m}+u_{n-1}^{m}-2 u_{n}^{m}\right),
\end{aligned}
$$

(41) and (42) are numerical expressions of the Newtonian fluid model.

\section{Numerical Results Analysis}

The time step was set as $\Delta t=0.001$ and the space step was set as $\Delta y=0.025$. The flow characteristics of the fractional $\mathrm{K}-\mathrm{BKZ}$ numerical model, the classical $\mathrm{K}-\mathrm{BKZ}$ numerical model, the fractional Maxwell numerical model, and the Newtonian fluid model were then compared.

5.1. Numerical Simulation of the Orifice. According to the initial conditions and the boundary conditions of (24), the numerical results of the flow velocity distribution of the start-up flow of the fractional K-BKZ numerical model, the classical K-BKZ numerical model, the fractional Maxwell numerical model, and the Newtonian fluid model in the orifice as the time increased have been shown in Figure 3. The basic parameters were $\alpha=0.8, \beta=0.3, \vartheta=0.2, a=0.2$. It can be seen from Figure 3 that the flow velocities of the four models in the orifice had a maximum value at $y=0.5$, and the velocity distribution curves were symmetric along the line where $y=0.5$, which conformed to the characteristics of the fluid flow in the pipe. By comparing the three-dimensional diagrams of the flow velocity distribution (Figures 3(a), 3(c), $3(\mathrm{e})$, and $3(\mathrm{~g}))$, it can be seen that when the time and the maximum velocity growth rate had the same value, the flow velocity distributions of the fractional K-BKZ numerical model and the classical K-BKZ numerical model had been gradually developing parabolic shapes. These were more rounded than the fractional Maxwell numerical model and the Newtonian fluid model, and the curvatures of the curves changed greatly. However, the variation trends of the fractional Maxwell numerical model and the Newtonian fluid model were not obvious. By comparing the three-dimensional flow velocity distributions of the fractional $\mathrm{K}-\mathrm{BKZ}$ numerical model and the classical K-BKZ numerical model (Figures 3(a) and 3(c)), it can be found that the curvature of the classical K-BKZ numerical model was larger than the fractional K-BKZ numerical model, and the former was more rounded than the latter. Figures 3(b), 3(d), 3(f), and $3(\mathrm{~h})$ show the two-dimensional flow velocity distributions of the four models with respect to time. It can be seen from Figures 3(b) and 3(d) that, as the time increased, the flow velocity distribution curves of the fractional K-BKZ numerical model and the classical K-BKZ numerical model gradually changed from a flat shape to a parabolic shape which fitted the flow characteristics of the actual viscoelastic fluid. Moreover, regardless of how large the maximum flow velocity was, the distribution curve always maintained good nonlinear characteristics and the curve's curvature was always more obvious than that of the fractional Maxwell numerical model and the Newtonian fluid model. This meant that the fluid that was simulated using the K-BKZ model (either the fractional K-BKZ numerical model or the classical K-BKZ numerical model) always maintained stronger viscoelasticity. However, the flow velocity distribution curve of the fractional Maxwell numerical model showed a flat shape at $t=0.5$. With the increase of time and the maximum velocity, the distribution curves gradually resembled that of the Newtonian fluid model, which meant that in the interval where $0<y<0.5$ or $0.5<y<1$, the flow velocity distribution curve of the Newtonian fluid model had been developing a straight shape and the curvature of the curve was basically unchanged. Besides, when time was large, the curve's curvature of the classical K-BKZ numerical model was larger than the fractional K-BKZ numerical model at the same time, which was consistent with the conclusions of the three-dimensional velocity distribution comparison.

Figure 4 shows the two-dimensional flow velocity distribution comparison of the fractional Maxwell numerical model and the Newtonian fluid model in the orifice at low velocity (that is, at short time) as the time increased. As can be seen from Figure 4, with the increase of time, the flow velocity distribution of the fractional Maxwell numerical model gradually changed from a flat shape to a parabolic shape, while the curvature of the curve declined compared with the previous time at $t=0.8$, and the fractional Maxwell numerical model gradually changed to the Newtonian fluid model. According to Figures 3 and 4, it can be found that the fractional Maxwell numerical model had good nonlinearity at low velocity or short time, while when the velocity was higher or the time was longer, it lost nonlinearity and viscoelasticity, which was consistent with the conclusions of studies [13] and [14].

Figure 5 shows the stresses variation of the fluid of four models at the center of the orifice. With the increase of the time, the stresses of the fractional Maxwell numerical model and the Newtonian fluid model increased linearly, but the stresses of the fractional K-BKZ numerical model and the classical K-BKZ numerical model increased slowly and tended to reach a steady state. The reason is that the K-BKZ model is able to effectively capture the phenomenon of shear thinning, while the Maxwell model and the Newtonian fluid model are not [14]. Since the classical K-BKZ numerical model has a damping function that converges to zero with time increasing, which can effectively describe the shear thinning phenomenon of viscoelastic fluid; therefore, the fractional K-BKZ numerical model derived from the model can also effectively capture the shear thinning phenomenon. Compared with the classical K-BKZ numerical model, the fractional K-BKZ numerical model employs the MittagLeffler function as the relaxation kernel instead of a powerlaw function. The Mittag-Leffler function is a generalized exponential function, which can improve the accuracy of the model [38], and it is more suitable to describe the relaxation process of polymer materials. The main component of the viscoelastic materials that were injected into the shock absorber was either methyl silicon rubber or methyl siloxane. These two materials belong to the class of high polymer 


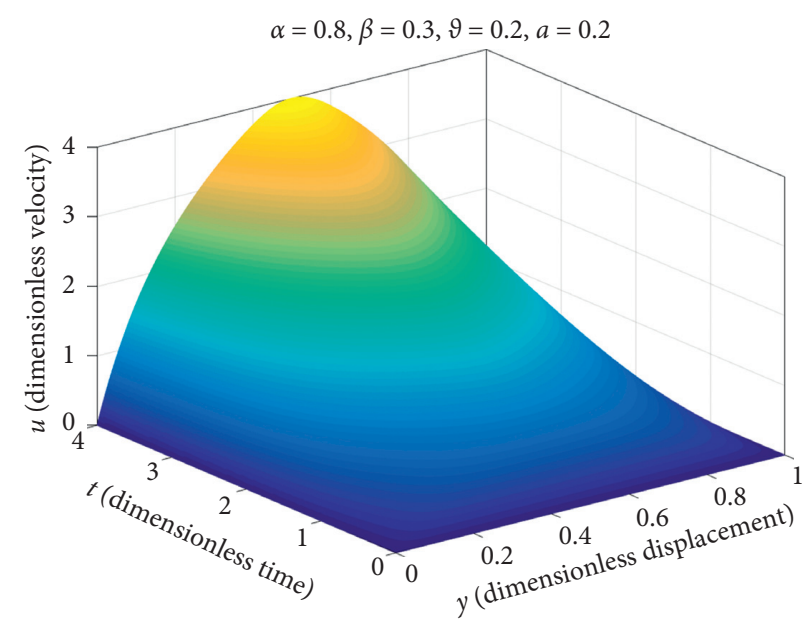

(a)

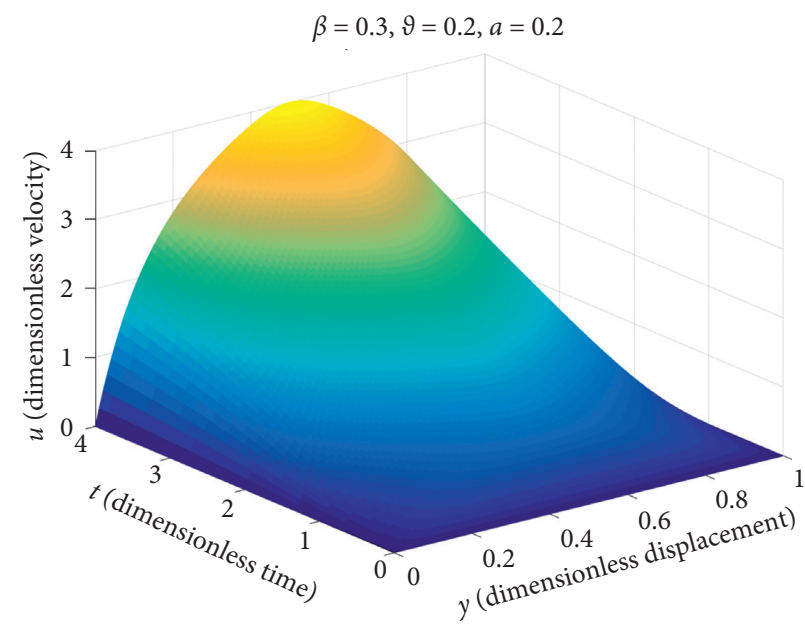

(c)

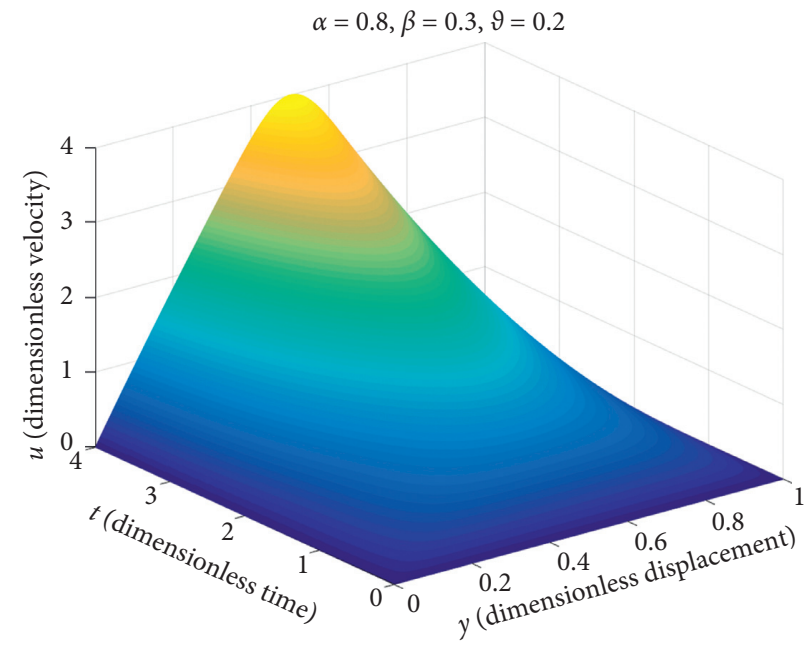

(e)

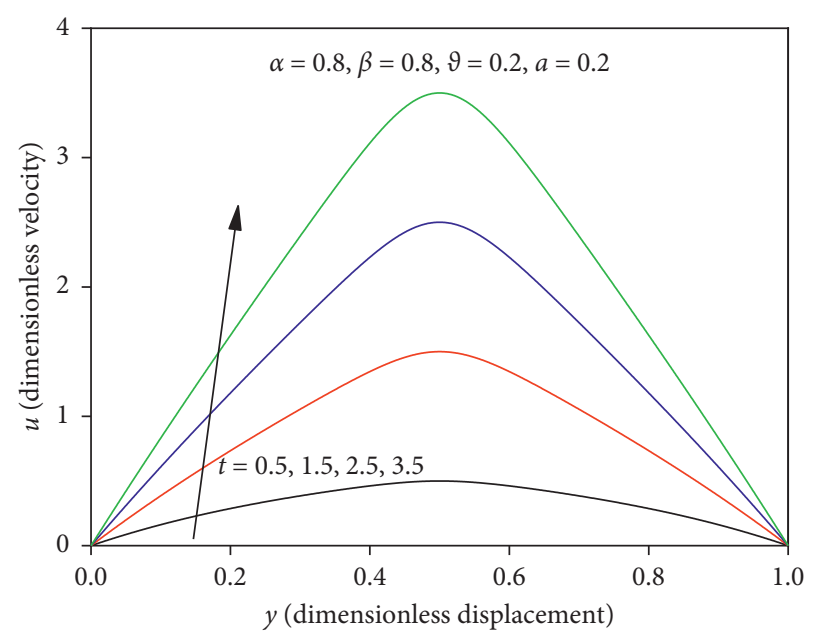

(b)

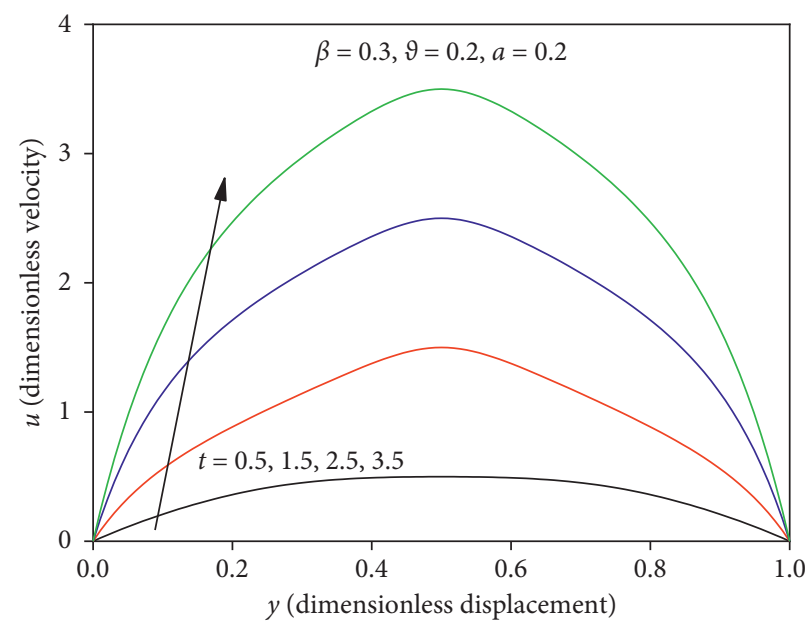

(d)

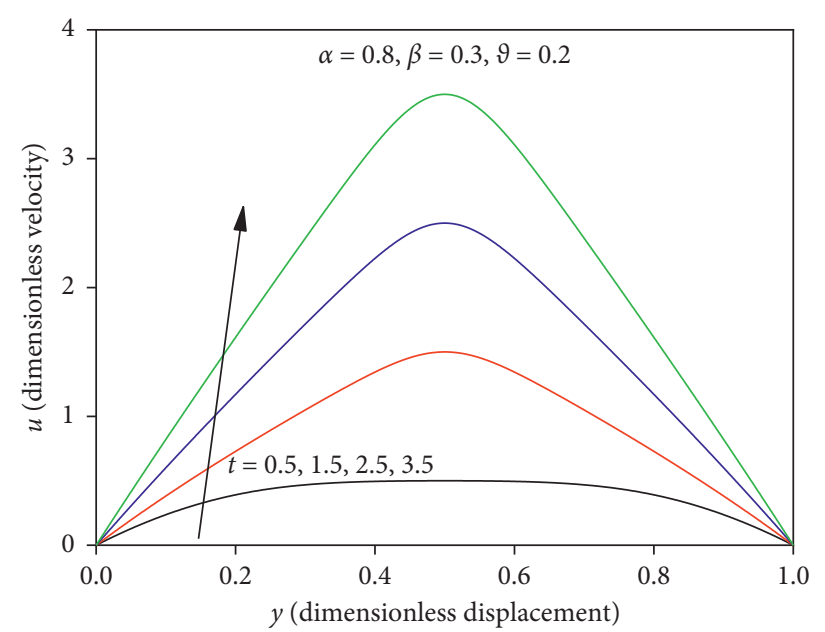

(f)

Figure 3: Continued. 


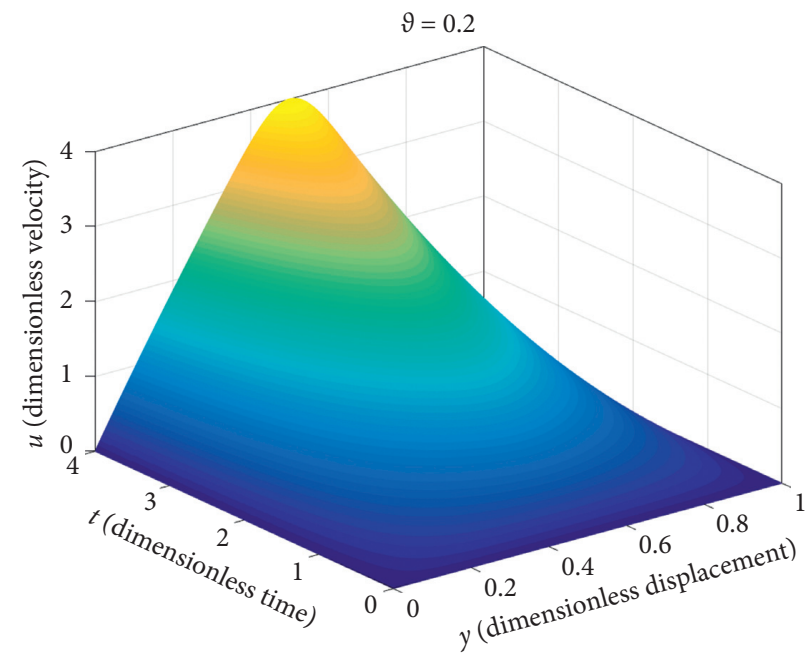

(g)

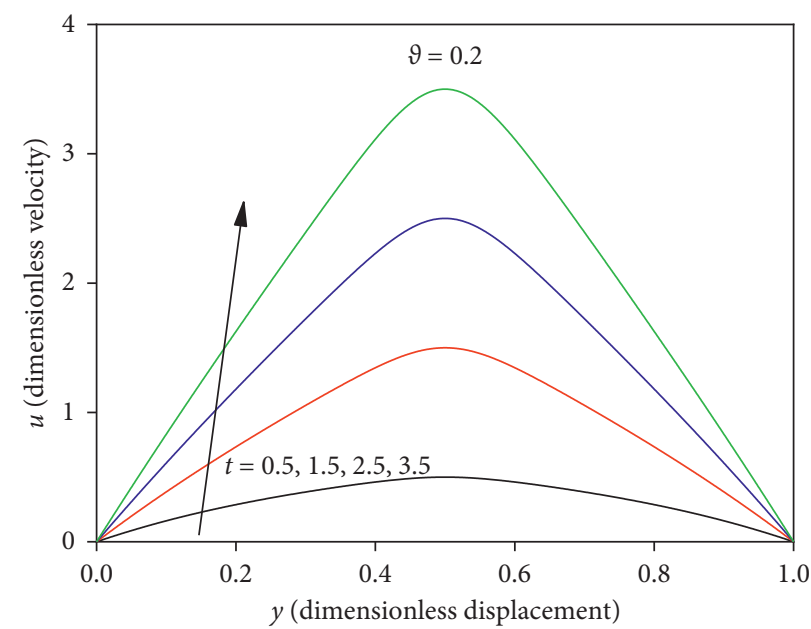

(h)

FIgURE 3: Flow velocity distributions of the four models in the orifice for different time values. (a) The three-dimensional flow velocity distribution of the fractional K-BKZ numerical model. (b) The two-dimensional flow velocity distribution of the fractional K-BKZ numerical model. (c) The three-dimensional flow velocity distribution of the classical K-BKZ numerical model. (d) The two-dimensional flow velocity distribution of the classical K-BKZ numerical model. (e) The three-dimensional flow velocity distribution of the fractional Maxwell numerical model. (f) The two-dimensional flow velocity distribution of the fractional Maxwell numerical model. (g) The three-dimensional flow velocity distribution of the Newtonian fluid model. (h) The two-dimensional flow velocity distribution of the Newtonian fluid model.

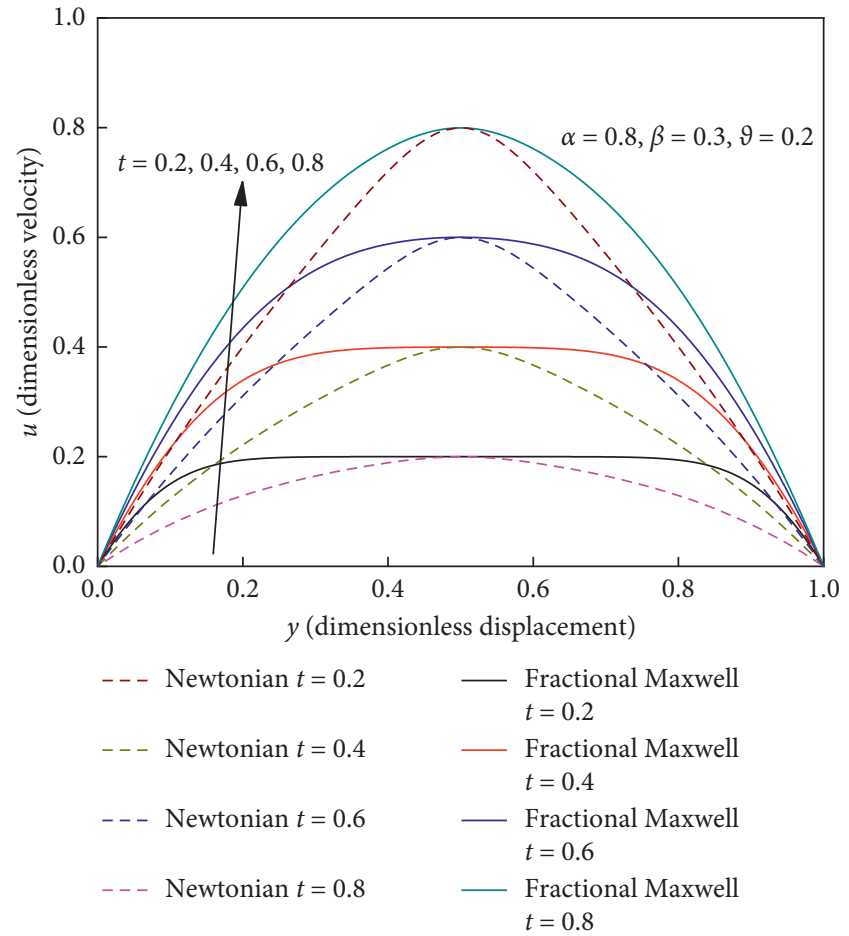

Figure 4: The two-dimensional flow velocity distribution comparison of the fractional Maxwell numerical model and the Newtonian fluid model in the orifice for different time values.

materials and pseudoplastic fluids, which possesses a shear thinning phenomenon. Therefore, the fractional $\mathrm{K}-\mathrm{BKZ}$ numerical model is able to capture the nonlinear viscoelastic properties of this viscoelastic material more accurately.
5.2. Numerical Simulation of the Gap. According to the initial conditions and the boundary conditions from (25), the flow velocity distributions of the start-up flow of four models in the gap of the shock absorber as the time increased have been shown in Figure 6. As can be seen from Figure 6, the flow velocity of the four models in the gap gradually decreased to zero from a certain value, which was consistent with the characteristics of the fluid flow between the plates. By comparing the three-dimensional diagrams of the flow velocity distribution of four models (Figures 6(a), 6(c), 6(e), and $6(\mathrm{~g}))$, it can be seen that when the time and the maximum velocity growth rate had the same value, the fractional K-BKZ numerical model and the classical K-BKZ numerical model showed a fuller velocity distribution and greater curvature than the fractional Maxwell numerical model and the Newtonian fluid model. Figures 6(b), 6(d), 6(f), and 6(h) show the two-dimensional flow velocity distributions of the four models varying with time. It can be seen from these graphs that the velocity distribution of the Newtonian fluid model was linear while the velocity distribution of the fractional K-BKZ numerical model maintained good nonlinearity as the time increased. When the time was short or the velocity was small, the classical K-BKZ numerical model and the fractional Maxwell numerical model were both similar to the fractional K-BKZ numerical model; all can accurately simulate the viscoelasticity of fluid. However, when the time was long or the velocity was large, the fractional Maxwell numerical model gradually developed to the Newtonian fluid model, while the classical K-BKZ numerical model lost the accuracy of viscoelasticity model. It can be seen in Figure 6(d) that the curves of $t=2.5$ and $t=3.5$ overlapped each other when $y>0.5$. The K-BKZ model with the relaxation kernel is described by power law function has 


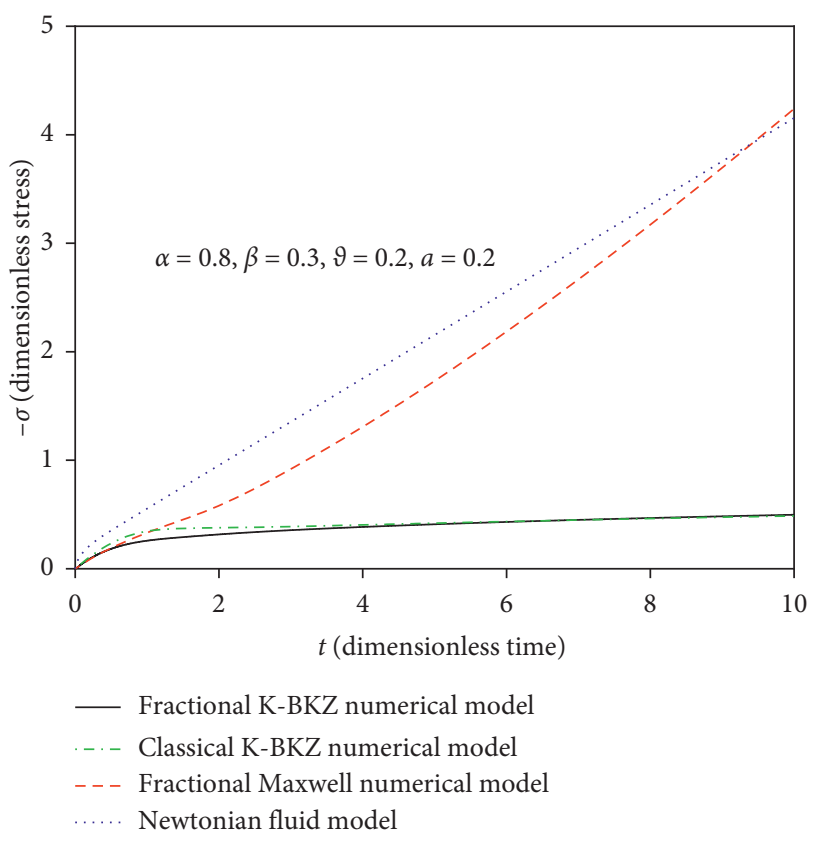

FIgURE 5: Stress distributions at the center point of the orifice.

the accuracy of the viscoelasticity model only at short times [14] and [26]. This phenomenon was proved in Figure 6, which is why the curve's curvature and shape of the classical $\mathrm{K}-\mathrm{BKZ}$ numerical model were larger and more rounded than the fractional $\mathrm{K}-\mathrm{BKZ}$ numerical model at the same time, when time was large.

Figure 7 shows the two-dimensional flow velocity distribution comparison of the fractional Maxwell numerical model and the Newtonian fluid model in the gap at low velocity or at short time as the time increased. As can be seen from Figure 7, with the increase of time, the flow velocity distribution of the Newtonian fluid model had a trend to develop to a linear shape, while the fractional Maxwell numerical model had a good nonlinearity. By combining Figures 6 and 7, it can be seen that at low velocity or short time, the fractional Maxwell numerical model had good nonlinearity, while the velocity is higher or the time is longer, it lost the nonlinearity and the viscoelasticity. The results further verified the simulation results of the start-up flow in the orifice: the fractional K-BKZ numerical model always keeps good nonlinearity and viscoelasticity as the time or the speed increased. However, the classical K-BKZ numerical model and the fractional Maxwell numerical model will respectively lose the viscoelastic accuracy and nonlinearity at long times. Besides, the Newtonian fluid model has no viscoelastic characteristics.

For the start-up flow in the gap, the stress distribution curves of four models near the side of the moving plate $(y=0)$ have been shown in Figure 8 . The stresses of the fractional Maxwell numerical model and the Newtonian fluid model increased linearly with the increasing time while the stresses of the fractional K-BKZ numerical model and the classical $\mathrm{K}-\mathrm{BKZ}$ numerical model gradually became steady, which was consistent with the increasing trend of the orifice. When $t=10$, the stress values of the start-up flow of the fractional Maxwell numerical model and the Newtonian fluid in the gap were 2.37 and 2.32, respectively, while the values of those two models in the orifice were 4.24 and 4.15 respectively. As can be seen from the values, the stresses of the start-up flow of the fractional Maxwell numerical model and the Newtonian fluid in the orifice all were 1.78 times that in the gap. The maximum velocity of the start-up flow in the orifice occurred at the center point $(y=0.5)$ while the maximum velocity of the start-up flow in the gap occurred near the side of the moving plate $(y=0)$, and there were also different boundary conditions. When the time step and the space step were the same, the calculated data of the velocity of the start-up flow in the orifice was half that of the data in the gap. As the stresses were calculated using the difference in velocity, and the stresses of the fractional Maxwell numerical model and the Newtonian fluid model increased linearly, the stresses of the start-up flow in the orifice were 1.78 times that in the gap. Although the velocity data could be kept consistent by reducing the number of steps, the adjustment of the steps would also cause a variation in the difference coefficient, so the result of the calculation would remain unchanged. The stresses of the start-up flow of the fractional K-BKZ numerical model and the classical K-BKZ numerical model in the orifice and the gap both were 0.50 and 0.48 , respectively. Due to the decrease in the difference of the data, the stresses were increased although the variations were not large. The reason was that the fractional $\mathrm{K}-\mathrm{BKZ}$ numerical model and the classical K-BKZ numerical model both had a damping function that converged to zero with the increase in the deformation, which made the models' stress tend to be a steady state. As a result, the variation in the stress of the fractional $\mathrm{K}-\mathrm{BKZ}$ numerical model was not large and the result was more stable; the fractional K-BKZ numerical model had a same characteristic with the K-BKZ model that it was able to effectively capture the shear thinning phenomenon.

\subsection{Influence Analyses of Parameters Change.} Figures 9-11 show the flow velocity distributions of the fractional K-BKZ numerical model and the classical K-BKZ numerical model in the gap for different parameters $a, \beta$, and $\vartheta$, respectively. By combining Figures $9-11$, we can find that when the time was short, the flow velocity distributions of the classical K-BKZ numerical model had a good consistency with the fractional K-BKZ numerical model, while when the time was large, the former was different from the latter. It can be seen from Figure 9 that as the time increased, no matter what the value of $a$ was, the fractional K-BKZ numerical model always maintained good viscoelasticity, while when $t=2.5$ and $t=3.5$, the curves of the classical K-BKZ numerical model overlapped each other. The viscoelastic accuracy of the classical K-BKZ numerical model was low at long times, and the accuracy did not change with the values of parameter $a$ increased. We can found from Figures 10 and 11 that the viscoelastic accuracies of the classical K-BKZ numerical model were strengthened with the increase of values of parameters $\beta$ and $\vartheta$, and the effect of parameter $\beta$ was greater than that of parameter $\vartheta$. Parameters $\beta$ and $\vartheta$ 


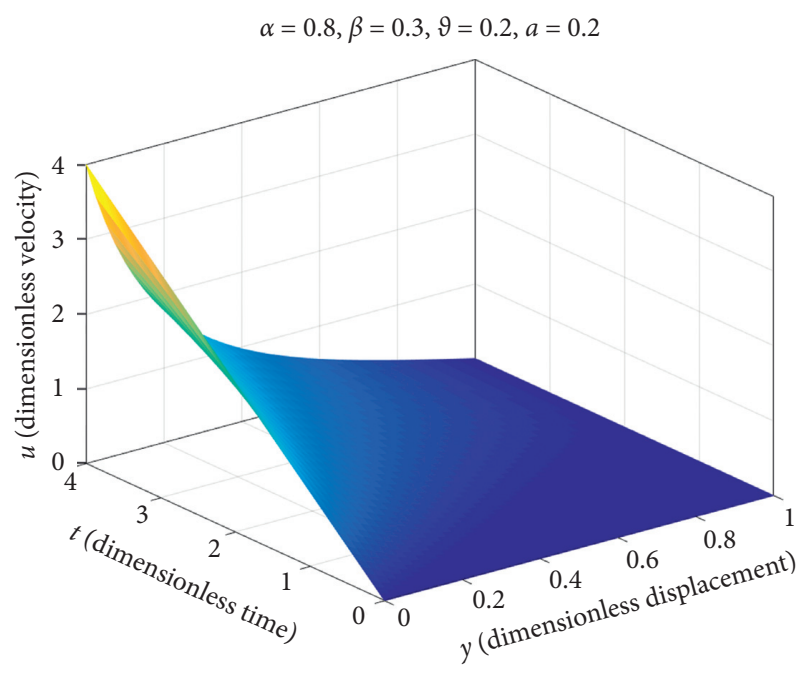

(a)

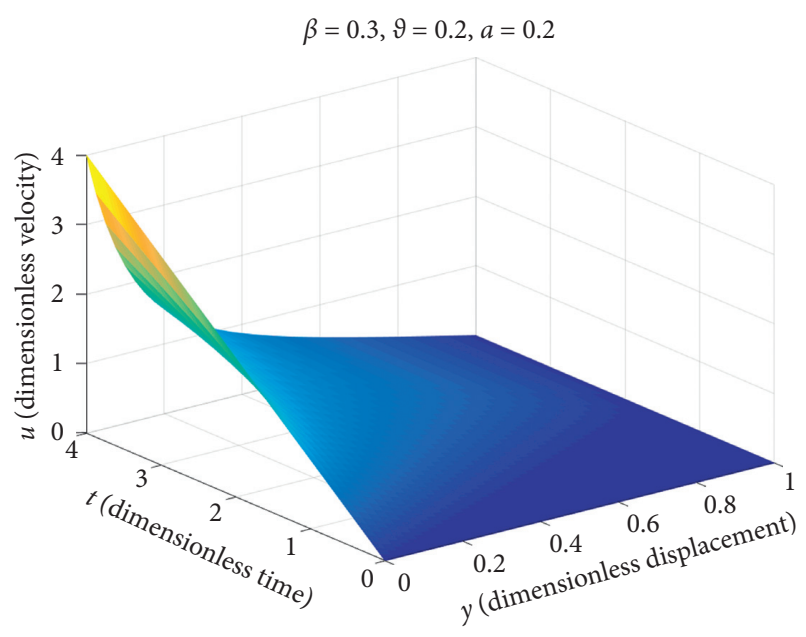

(c)

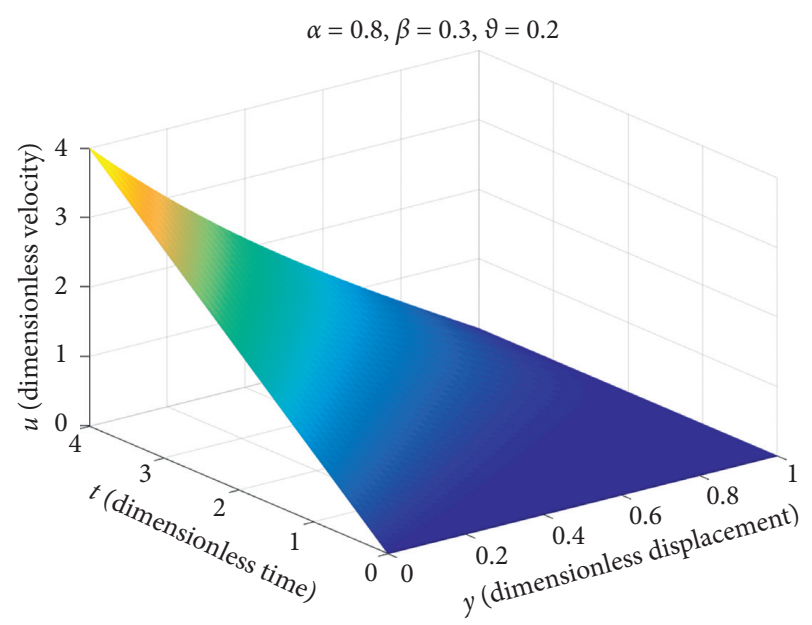

(e)

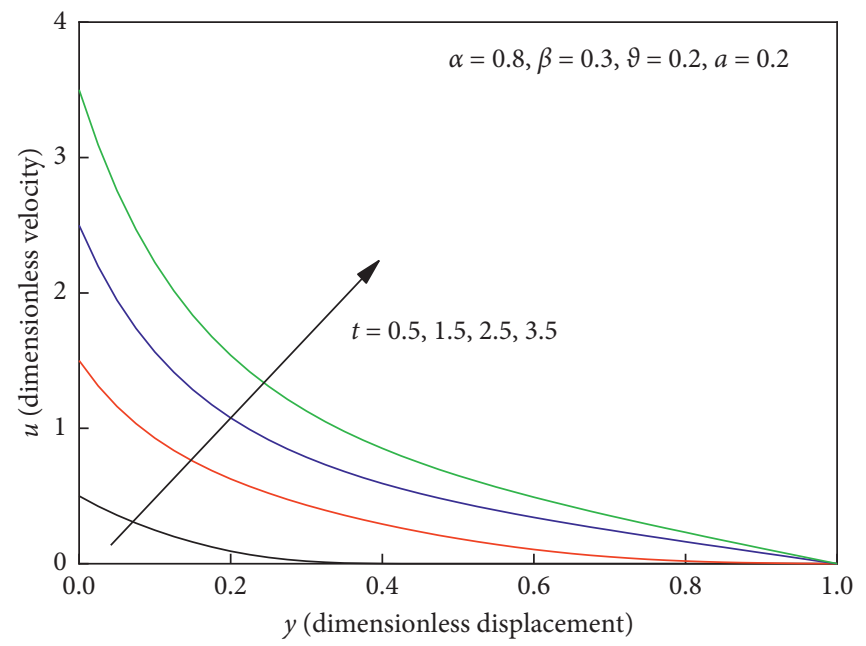

(b)

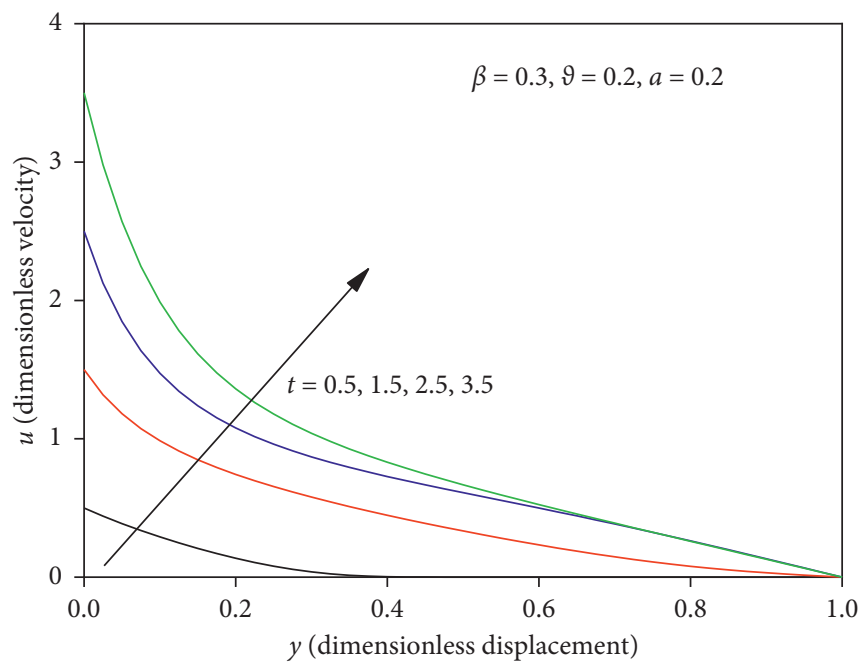

(d)

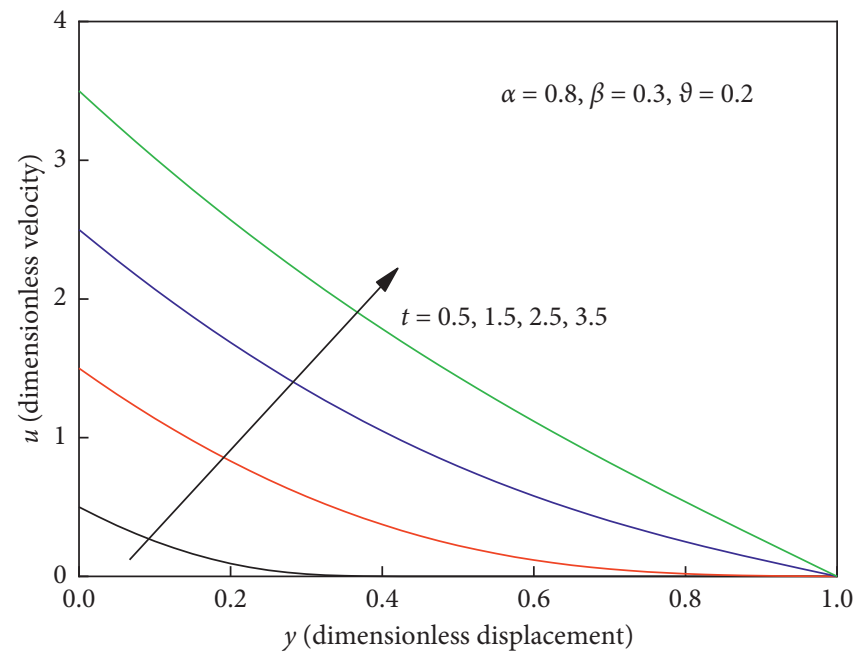

(f)

Figure 6: Continued. 


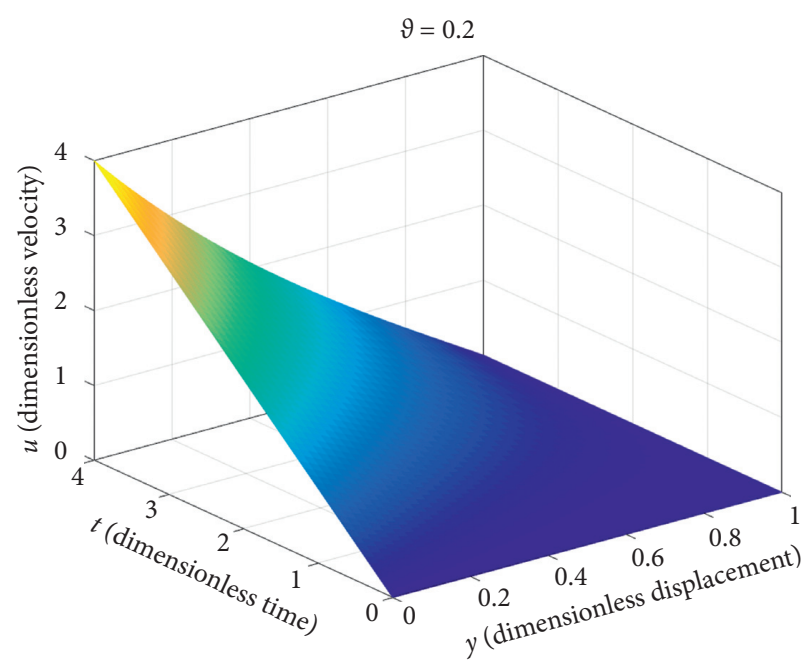

(g)

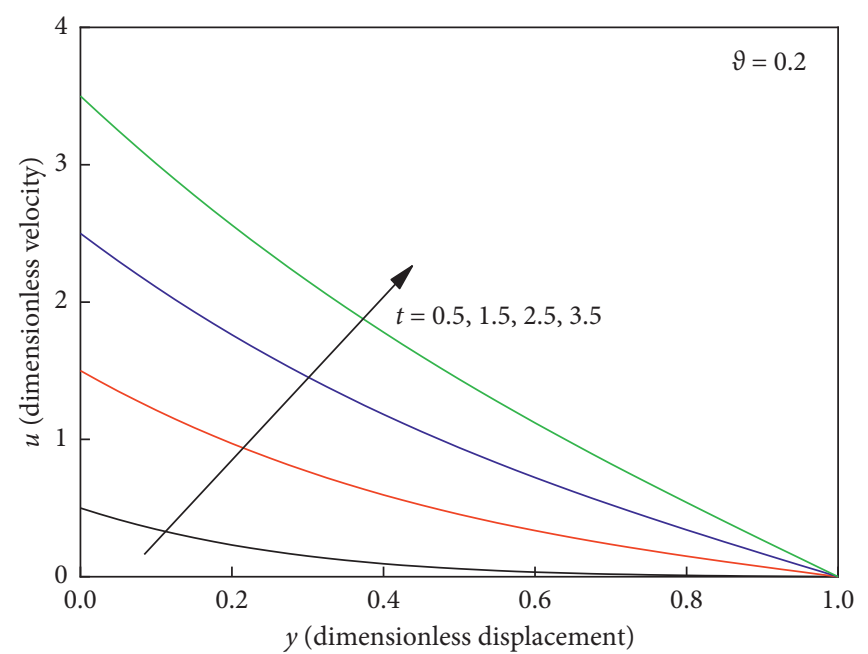

(h)

Figure 6: Flow velocity distributions of the four models in the gap for different time values. (a) The three-dimensional flow velocity distribution of the fractional K-BKZ numerical model. (b) The two-dimensional flow velocity distribution of the fractional K-BKZ numerical model. (c) The three-dimensional flow velocity distribution of the classical K-BKZ numerical model. (d) The two-dimensional flow velocity distribution of the classical K-BKZ numerical model. (e) The three-dimensional flow velocity distribution of the fractional Maxwell numerical model. (f) The two-dimensional flow velocity distribution of the fractional Maxwell numerical model. (g) The three-dimensional flow velocity distribution of the Newtonian fluid model. (h) The two-dimensional flow velocity distribution of the Newtonian fluid model.

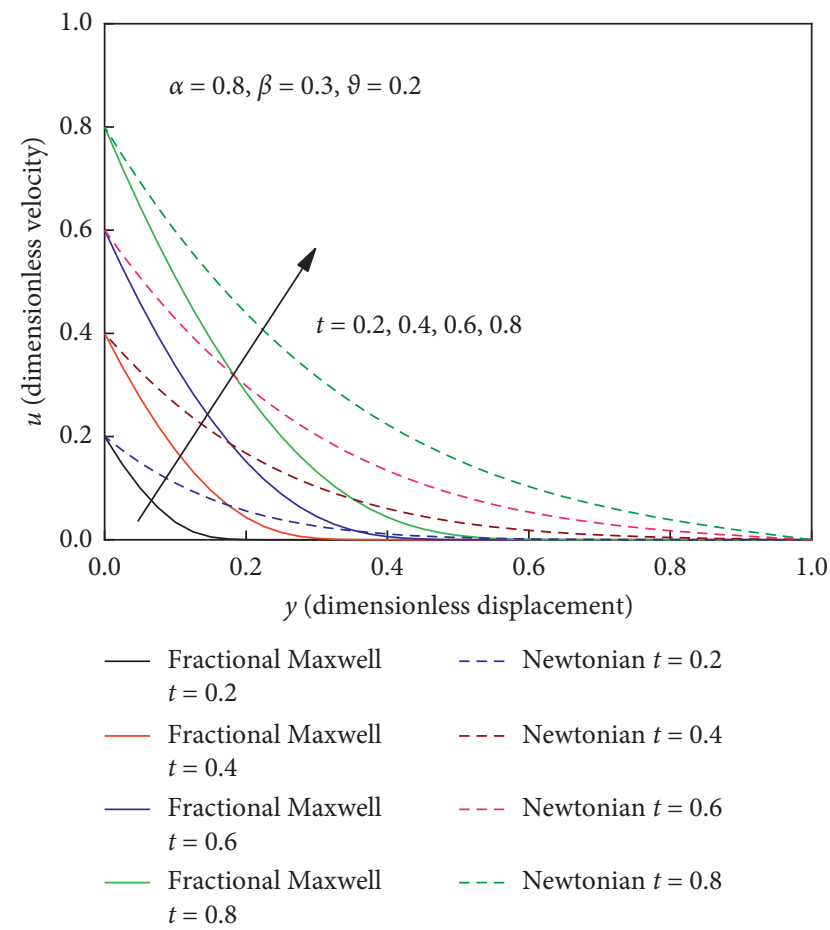

Figure 7: The two-dimensional flow velocity distribution comparison of the fractional Maxwell numerical model and the Newtonian fluid model in the gap for different time values.

were the greater, the accuracy of the classical K-BKZ numerical model was the better. Comparatively, the fractional K-BKZ numerical model had good accuracy.

The flow velocity distributions of the fractional K-BKZ numerical model with change of parameters are shown in

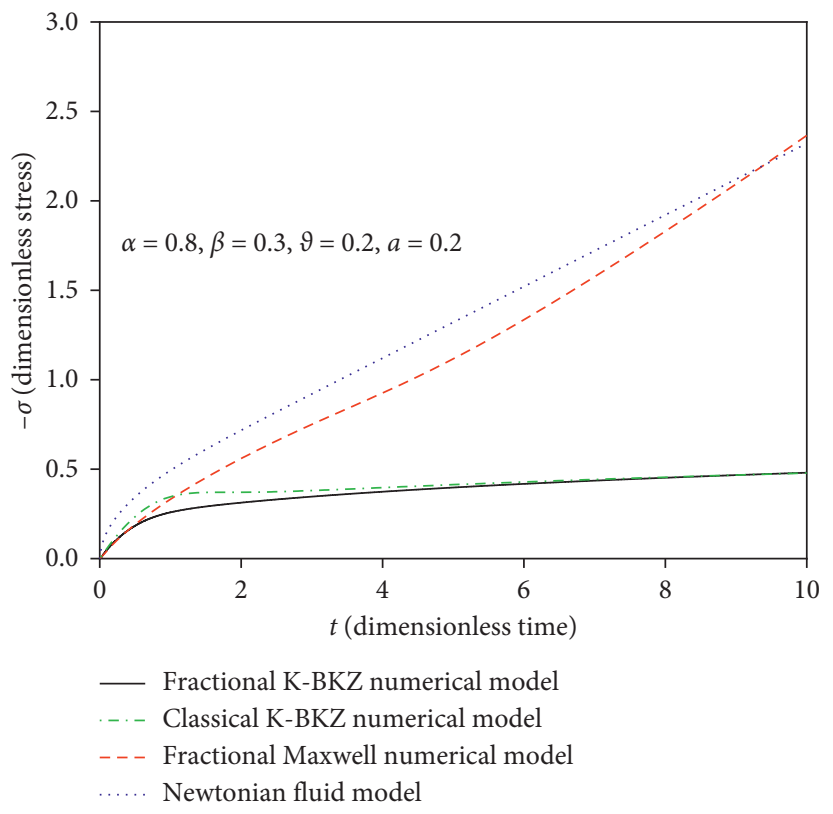

Figure 8: Stress distribution of four models near the side of the moving plate in the gap.

Figure 12. With the increase of the parameter $a$, the nonlinearity and the flow velocity of the fractional $\mathrm{K}-\mathrm{BKZ}$ numerical model gradually increased (as shown in Figure 7). The parameter $a$ is related with the damping function [28]. The less the parameter $a$ is, the more the damping function tends to zero and the larger the viscosity decreases. Because of the damping function tends to zero, the molecular chain entanglement and network structure of the viscoelastic material are mostly destroyed [28]. The parameters $\alpha$ and $\beta$ 


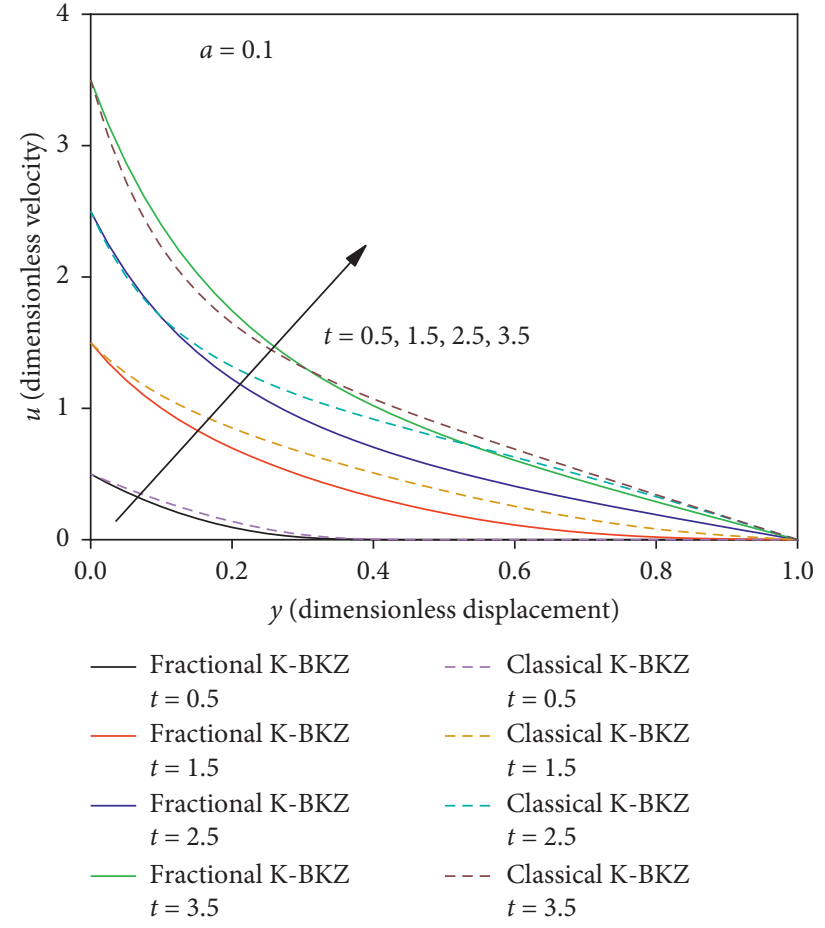

(a)

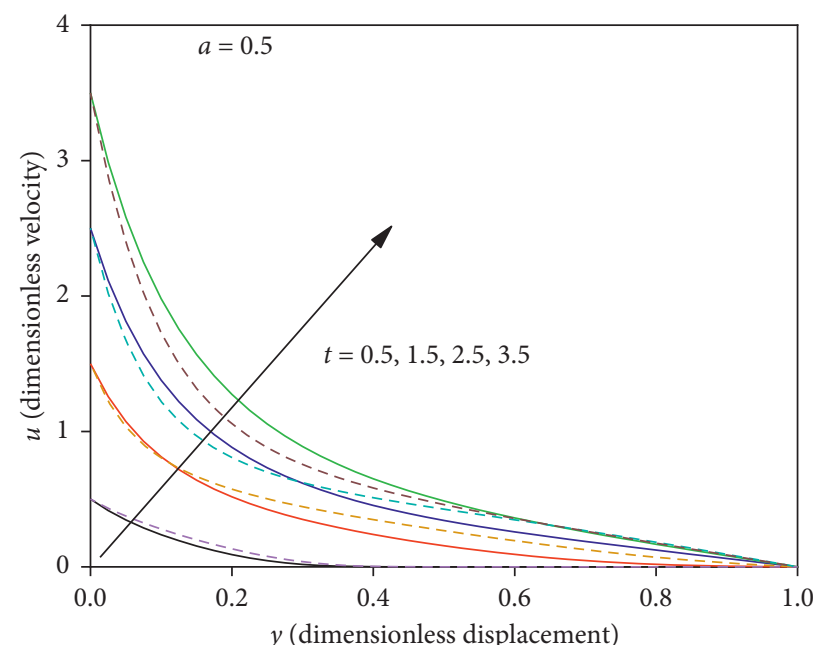

(c)

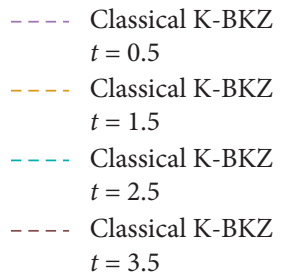

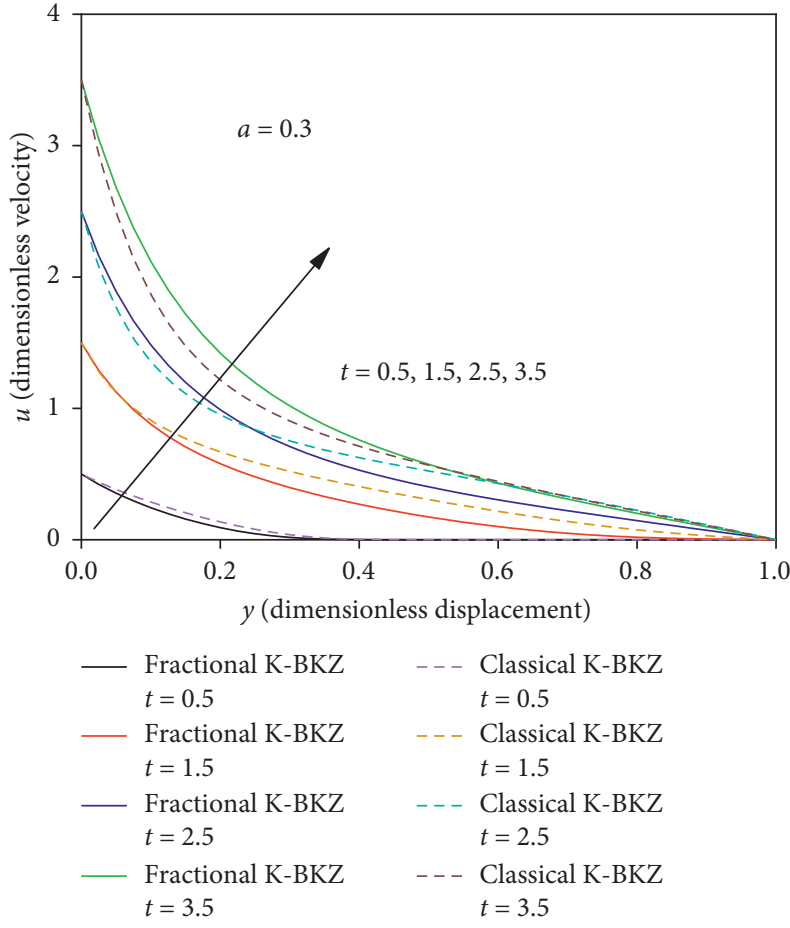

(b)

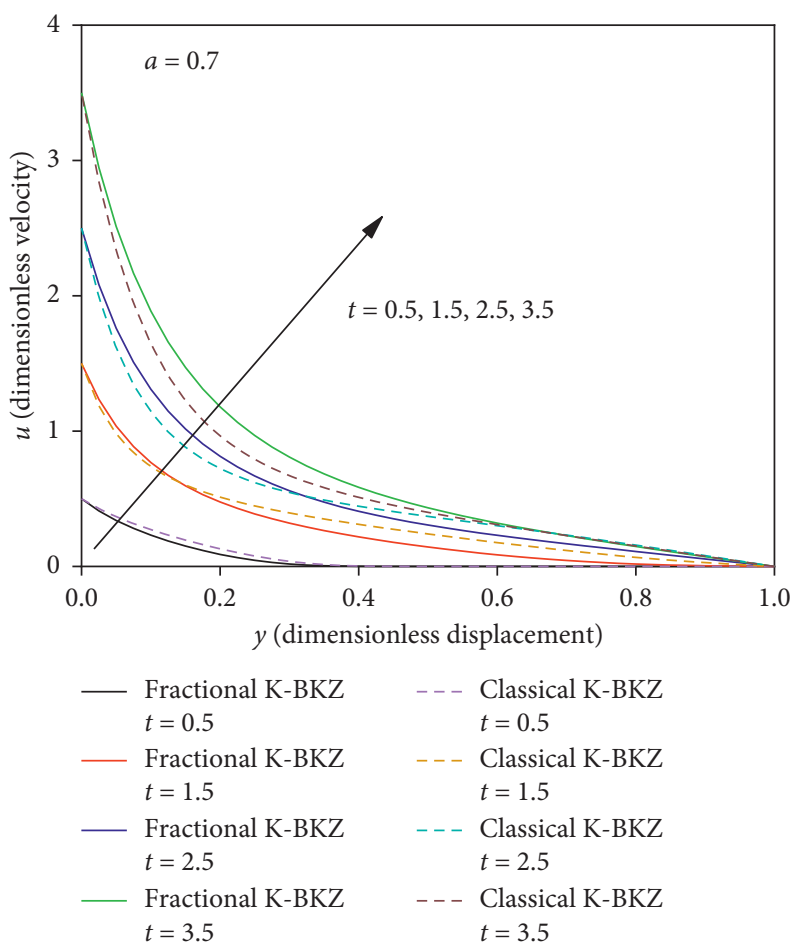

(d)

FIgURE 9: Flow velocity distributions of the fractional K-BKZ numerical model and classical K-BKZ numerical model in the gap for parameter $a$ : (a) $a=0.1$; (b) $a=0.3$; (c) $a=0.5$; (d) $a=0.7$.

were the fractional orders. It can be seen from Figure 7 that as $\alpha$ and $\beta$ increased, the trends of the flow velocity decreased and the curvature of the flow velocity distribution curve gradually decreased, so the viscosity decreased. As shown in
Figure 7 that the dependence of the parameter $\zeta$ on the flow velocity field had the same trend as that of $\alpha$ and $\beta$. The parameter $\zeta$ was smaller, the velocity curve curvature was larger, and the nonlinearity was stronger. 

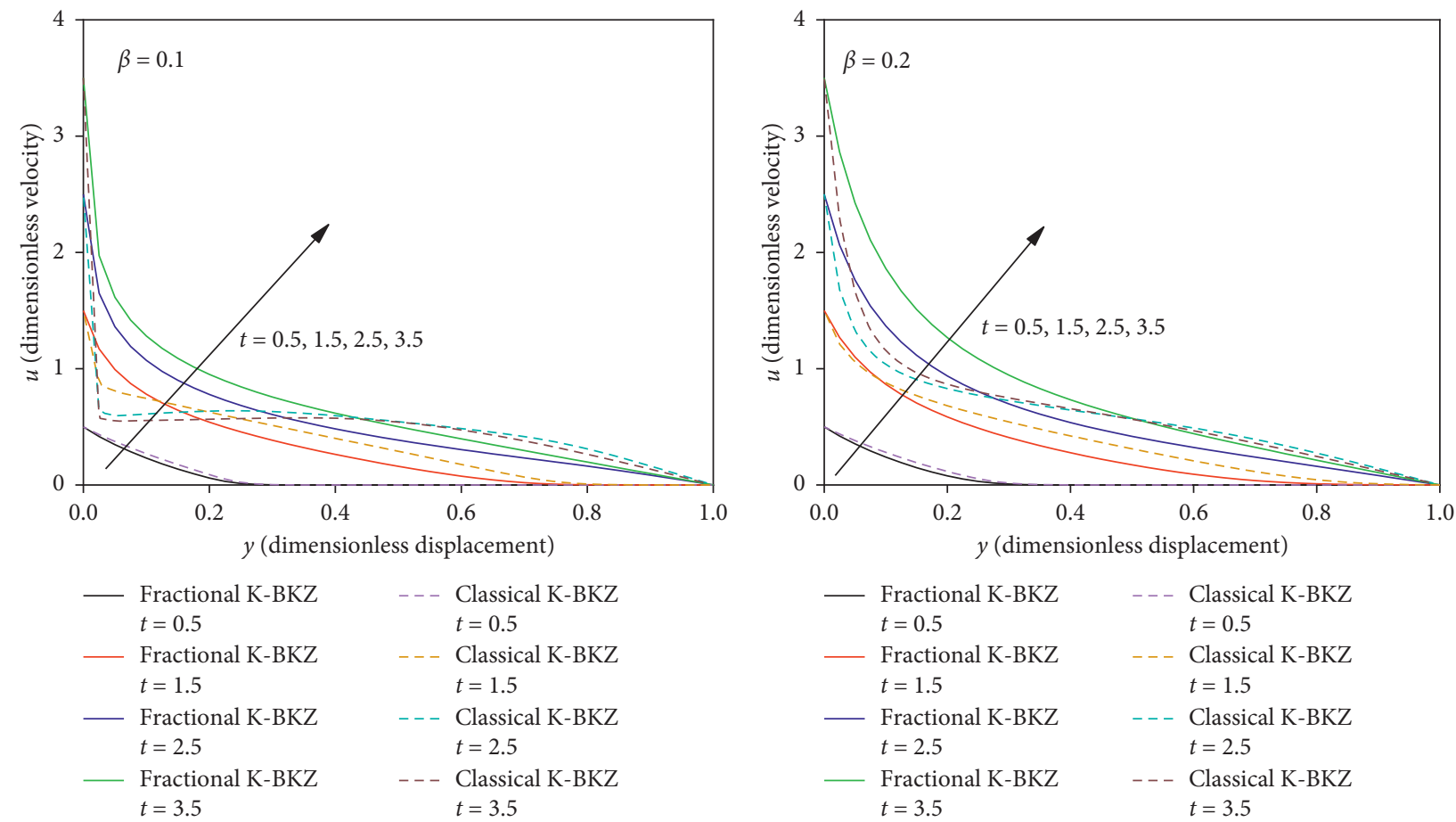

(a)

$$
\begin{aligned}
- \text { - } & \text { Classical K-BKZ } \\
& t=0.5 \\
--- & \text { Classical K-BKZ } \\
& t=1.5 \\
--- & \text { Classical K-BKZ } \\
& t=2.5 \\
--- & \text { Classical K-BKZ } \\
& t=3.5
\end{aligned}
$$

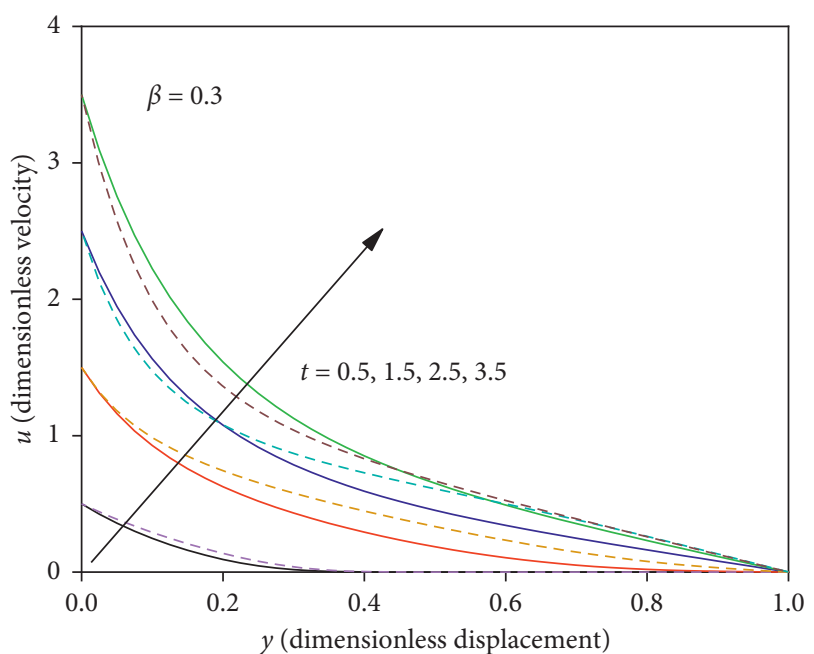

(c)

$$
\begin{aligned}
-- \text { - } & \text { Classical K-BKZ } \\
& t=0.5 \\
--- & \text { Classical K-BKZ } \\
& t=1.5 \\
--- & \text { Classical K-BKZ } \\
& t=2.5 \\
--- & \text { Classical K-BKZ } \\
& t=3.5
\end{aligned}
$$

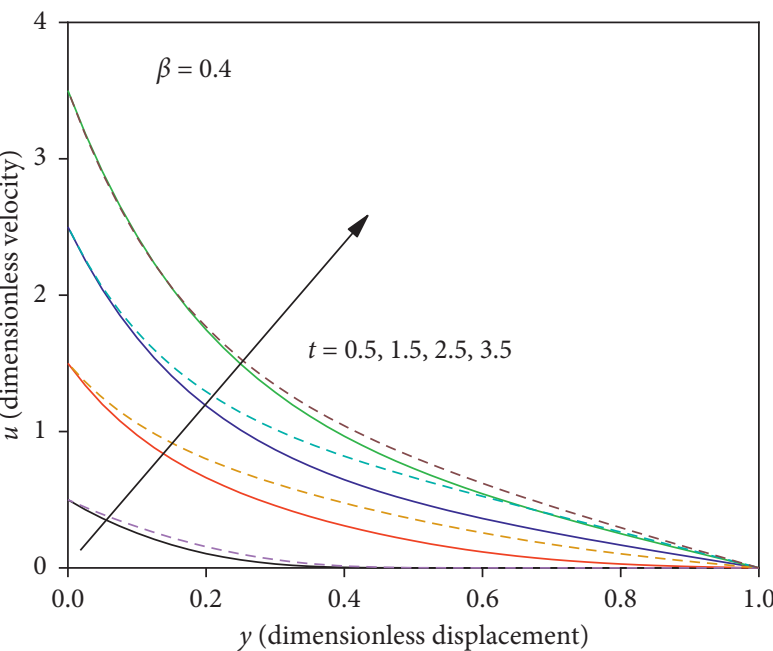

$$
\begin{array}{lrl}
- \text { Fractional K-BKZ } & --- & \text { Classical K-BKZ } \\
t=0.5 & t=0.5 \\
\text { Fractional K-BKZ } & --- & \text { Classical K-BKZ } \\
t=1.5 & t=1.5 \\
\text { Fractional K-BKZ } & --- & \text { Classical K-BKZ } \\
t=2.5 & & t=2.5 \\
\text { Fractional K-BKZ } & --- & \text { Classical K-BKZ } \\
t=3.5 & t=3.5
\end{array}
$$

(d)

FIgURE 10: Flow velocity distributions of the fractional K-BKZ numerical model and classical K-BKZ numerical model in the gap for parameter $\beta$ : (a) $\beta=0.1$; (b) $\beta=0.2$; (c) $\beta=0.3$; (d) $\beta=0.4$.

\section{Test Verification}

The test system consisted of an AC servo motor, a machine controller, a shock absorber made from a glass tube, a high- speed camera, image acquisition software, and a spotlight (as shown in Figure 13(a)). To facilitate the capture of the startup flow process of the viscoelastic fluid, the plastic tube was used as the piston and the acrylic glass tube was used as the 

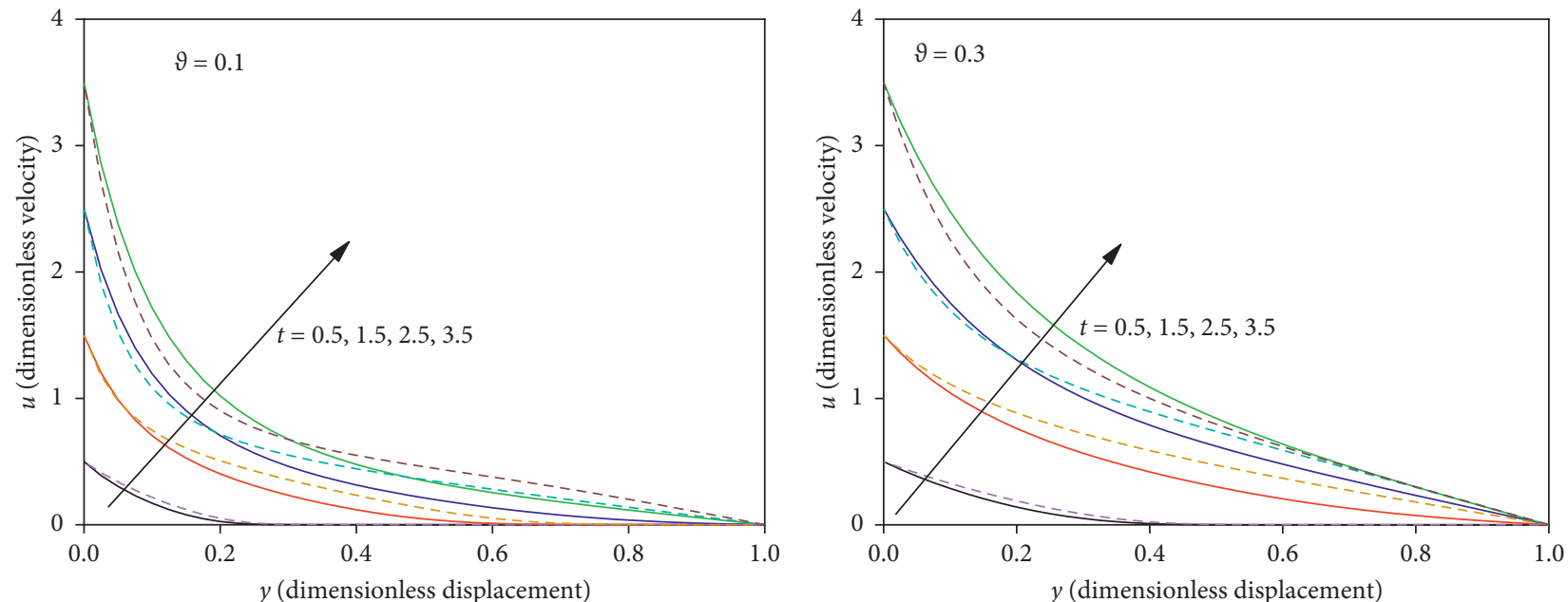

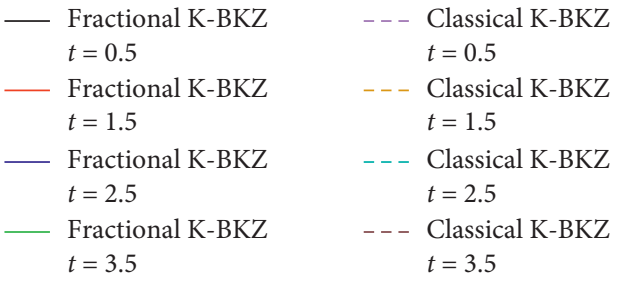

(a)

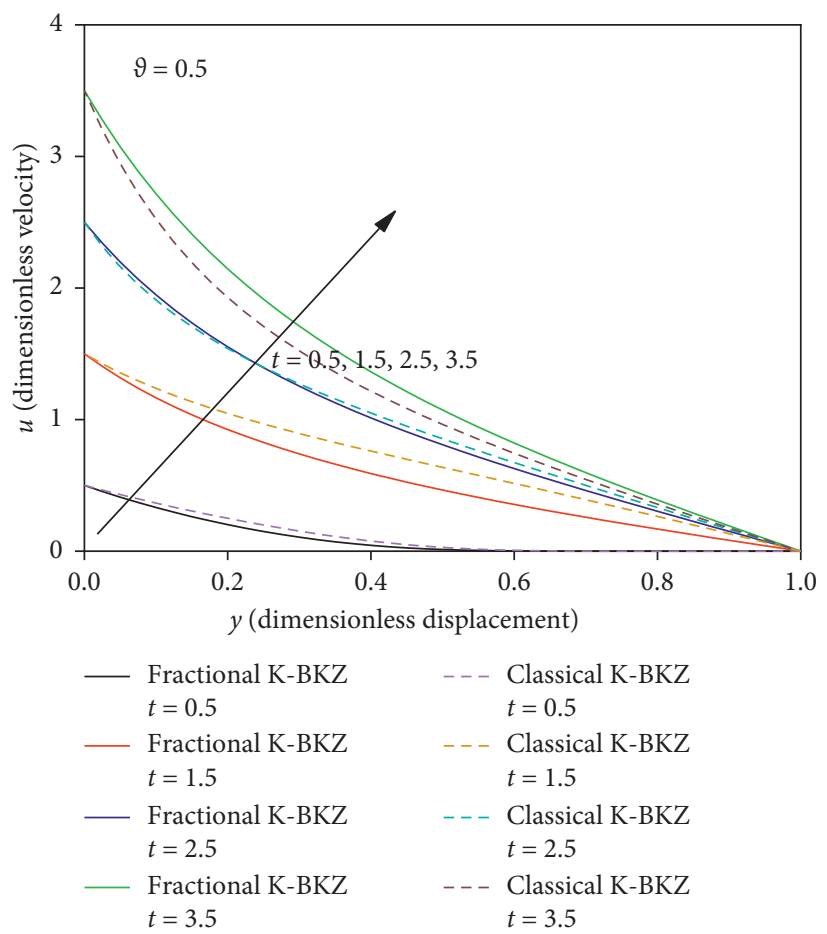

(c)

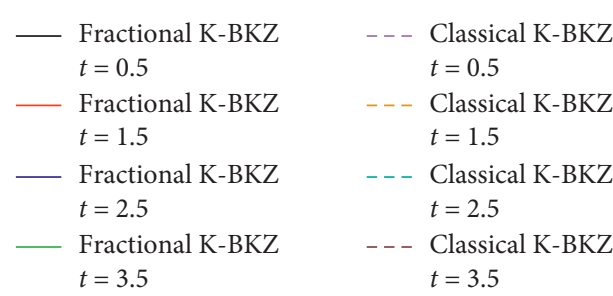

(b)

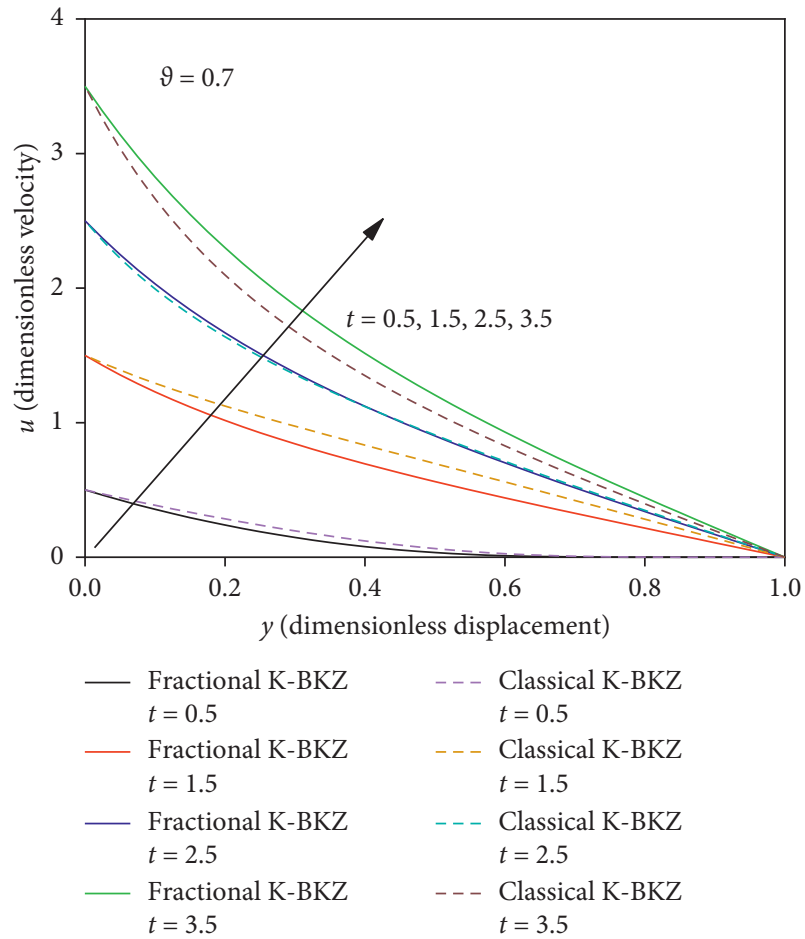

(d)

Figure 11: Flow velocity distributions of the fractional K-BKZ numerical model and classical K-BKZ numerical model in the gap for parameter $\vartheta$ : (a) $\vartheta=0.1$; (b) $\vartheta=0.3$; (c) $\vartheta=0.5$; (d) $\vartheta=0.7$.

shock absorber's chamber (as shown in Figure 13(b)). The test device was designed according to the actual sizes of the shock absorber's piston and orifice. The chamber's inner diameter was $20 \mathrm{~mm}$, the thickness of the piston was $20 \mathrm{~mm}$, and the diameter of orifice was $1.5 \mathrm{~mm}$. In order to facilitate the process of capturing the flow of the orifice, the gap 


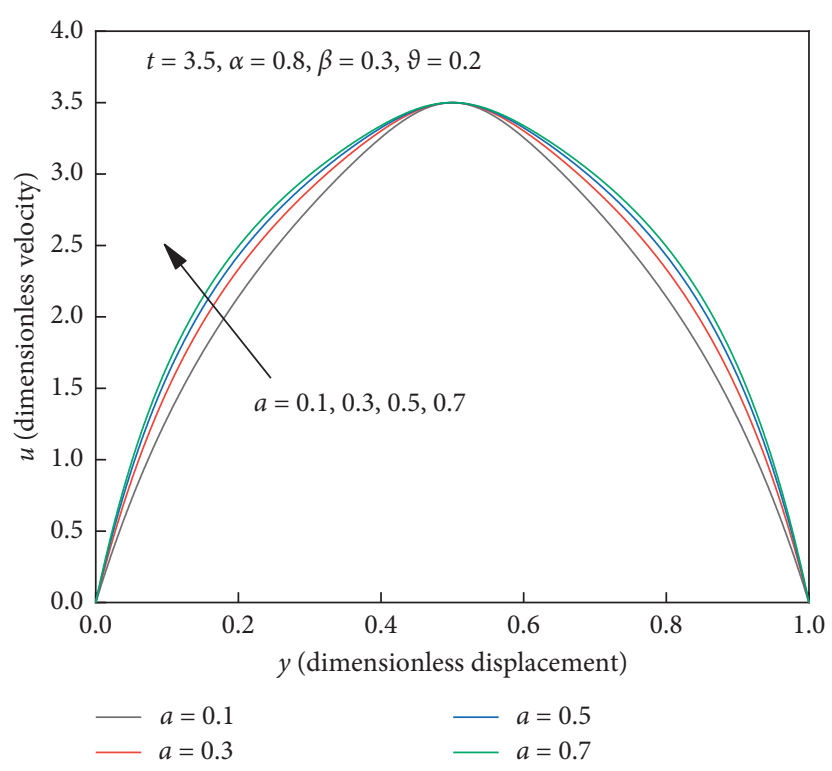

(a)

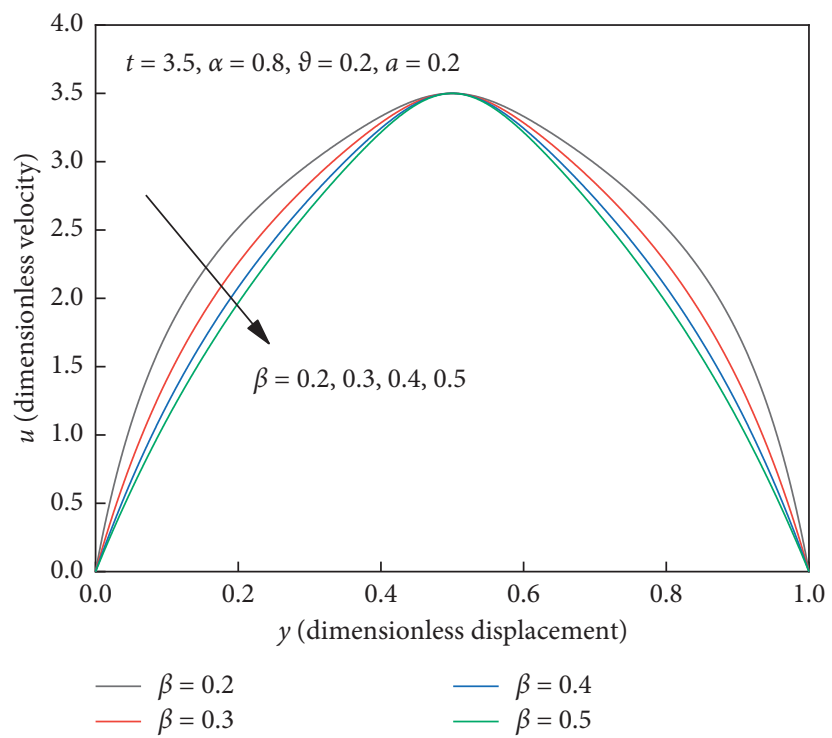

(c)

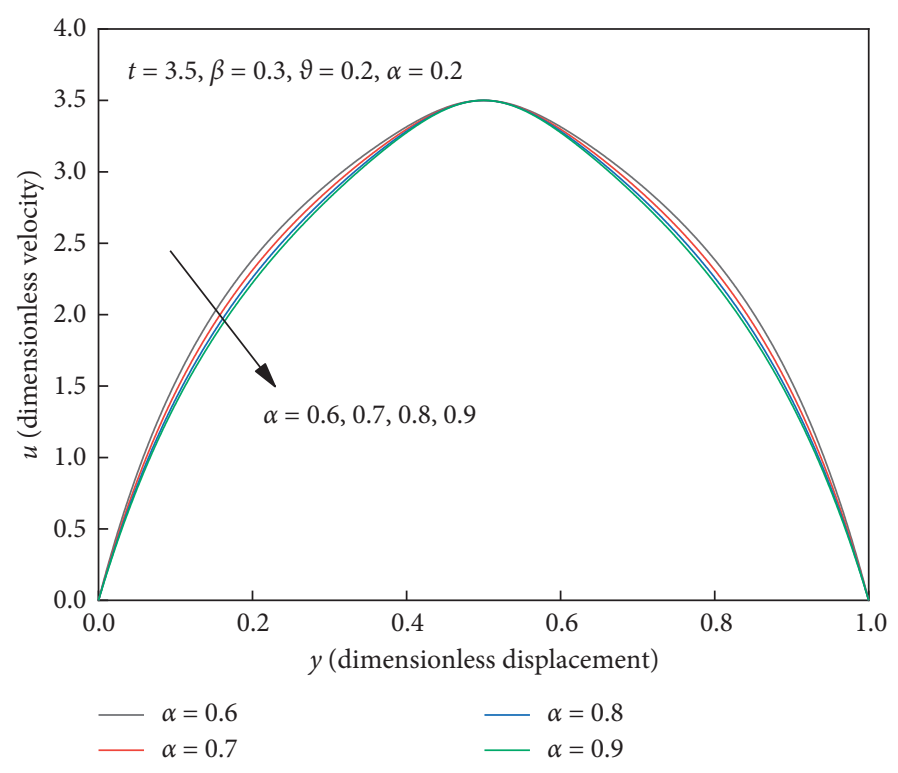

(b)

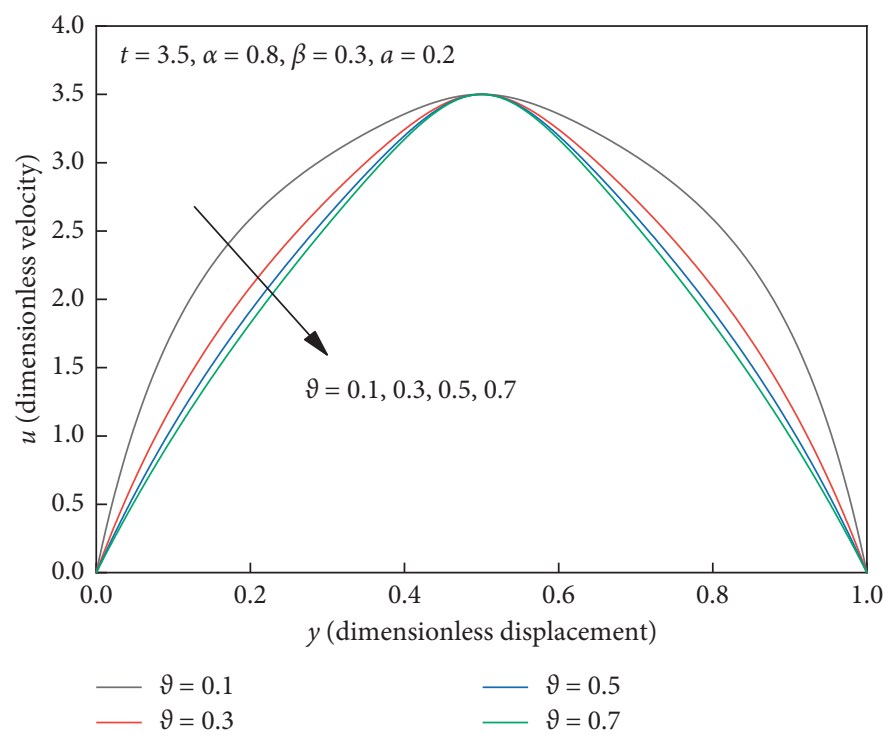

(d)

FIGURE 12: Flow velocity distributions of the orifice of the fractional K-BKZ numerical model for different parameters. (a) Parameter a. (b) Parameter $\alpha$. (c) Parameter $\beta$. (d) Parameter $\vartheta$.

between the piston and the chamber was removed and a sealing ring was installed on the head of the plastic piston tube in order to prevent the viscoelastic fluid from squeezing into the gap during motion.

A Mitsubishi AC servo motor was used as the motion source to drive the piston. The motor controller was used to adjust the rotational speed to keep the piston at a constant speed or acceleration. A Fastec HiSpec 5 high-speed camera and the accompanying image acquisition software were used to capture the flow condition of the viscoelastic fluid in the orifice at the start-up of the piston. In the shooting process, the spotlight was used as an auxiliary light source to enhance the camera's image capture.
A viscoelastic material, with methylsiloxane as the main component with a dynamic viscosity of $5 \times 10^{5} \mathrm{cSt}$ and a density of $1.1 \mathrm{~g} / \mathrm{cm}^{3}$, was injected into the chamber of the shock absorber in the glass tube. The motor controller was adjusted to start the piston from $0 \mathrm{~mm} / \mathrm{s}$, and the acceleration was maintained at $1.0 \mathrm{~mm} / \mathrm{s}^{2}$. The experiment was carried out at ambient temperature. The acquisition frequency of the high-speed camera was 500 frames per second, so it could record the flow conditions of the viscoelastic fluid at different moments, as shown in Figure 14. The black region in the figure shows the viscoelastic material while the white region is the piston of the plastic tube. During the experiment, the auxiliary light 


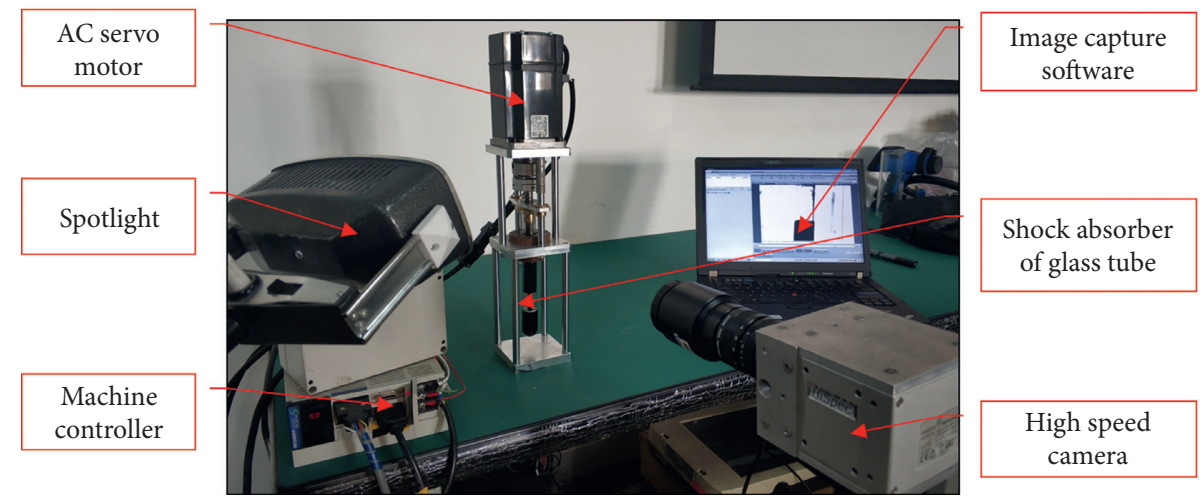

(a)

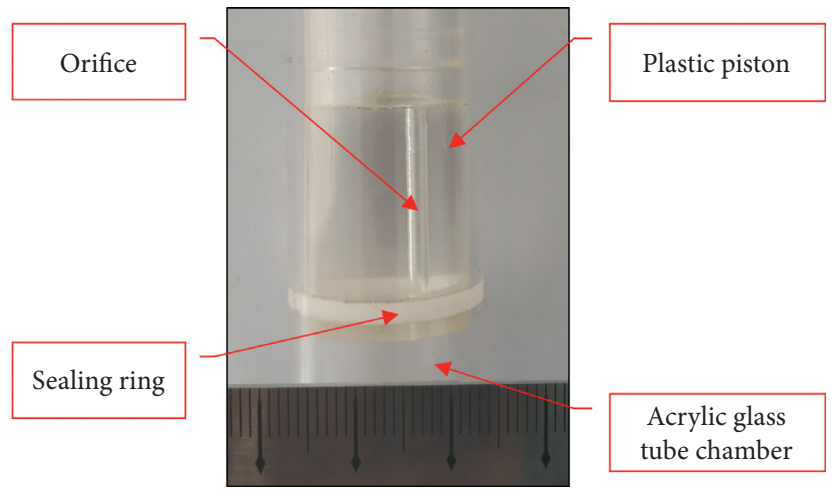

(b)

FIgURE 13: The test system for the start-up flow measure. (a) The test system layout for the start-up flow measurement. (b) The partial enlarged picture of the glass tube shock absorber.

source was on the left side of the shock absorber in the glass tube so that the reflective point was easily visible at the left half when the high-speed camera was shooting. As a result, some areas on the left side of the test results were missing and became white due to overexposure. Due to the small acceleration, the fluid velocity was also small in the first $0.6 \mathrm{~s}$, and the change in the viscoelastic material was not obvious.

According to the test results, the flow velocity was the largest at the orifice's center and was smaller around the orifice's edge. As the time increased and the piston's speed increased, the shape of the viscoelastic material gradually changed from a flat shape to a parabolic shape. The start-up flow in the orifice always maintained stronger viscoelasticity during the flow process.

The velocity-time variation of the start-up flow in the orifice was obtained by substituting the model's parameters listed in Table 1 into the fractional K-BKZ numerical model. The fluid level distribution curves of the fractional $\mathrm{K}-\mathrm{BKZ}$ numerical model with increasing time were obtained by integration of the velocity, as shown in Figure 15. The shape of the fluid level distribution, simulated by the fractional K-BKZ numerical model, gradually changed from a flat shape to a parabolic shape as the time increased, which was basically the same as the trend of the test results.

$$
\begin{aligned}
& \kappa \approx \frac{R(t)}{\lambda^{-\alpha}} \Gamma(1-\alpha), \\
& \psi \approx \frac{R(t)}{\lambda^{-\beta}} \Gamma(1-\beta) .
\end{aligned}
$$

The measurement method of the contact angle used in the literature [40] was applied in this paper, and the test results were combined for modification. As an example from the test results of the viscoelastic material's fluid level at $t=1.0 \mathrm{~s}$, two parameters of the maximum fluid level $H$ and the contact angle $\gamma$ were proposed, as shown in Figure 16. The maximum fluid level was taken as the vertical distance from the horizontal line of the lowest fluid level (i.e., the initial position of the viscoelastic fluid) to the highest point at the chosen time. This could verify the consistency of the numerical model results and the test results at the same time. An auxiliary tangent was drawn from the lowest point of the fluid level. The contact angle was the included angle between the auxiliary tangent and the horizontal line of the lowest fluid level, which was mainly used to investigate the viscoelasticity of the fluid. Some areas on the left side of the test results become white due to overexposure, so the contact angles were subjected to measurement on the right. According to the scale length, the maximum fluid level and the contact angle of the 


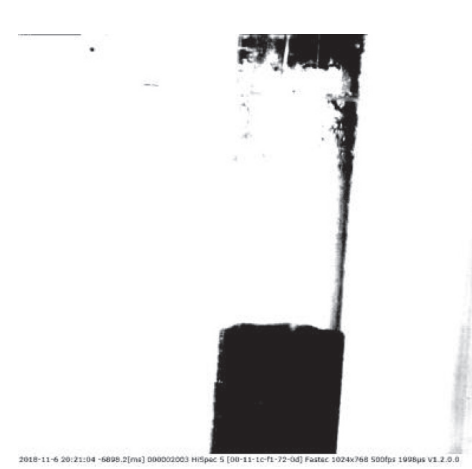

(a)

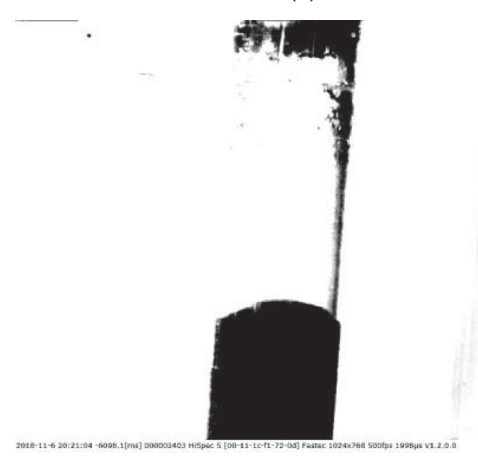

(c)

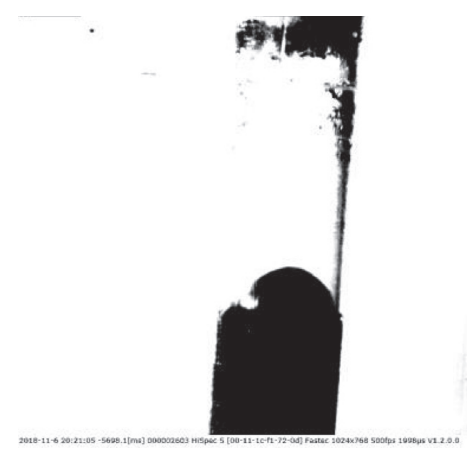

(e)
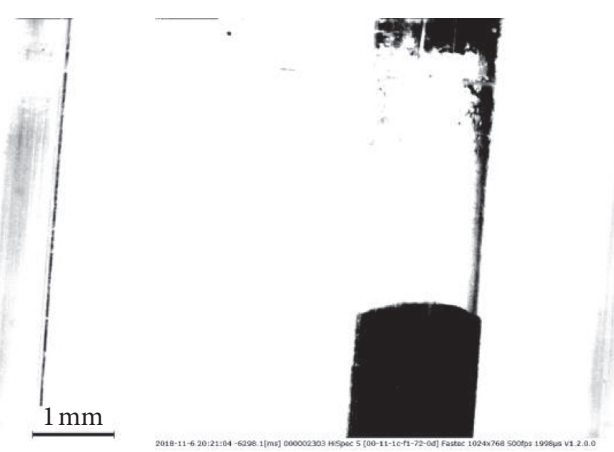

(b)
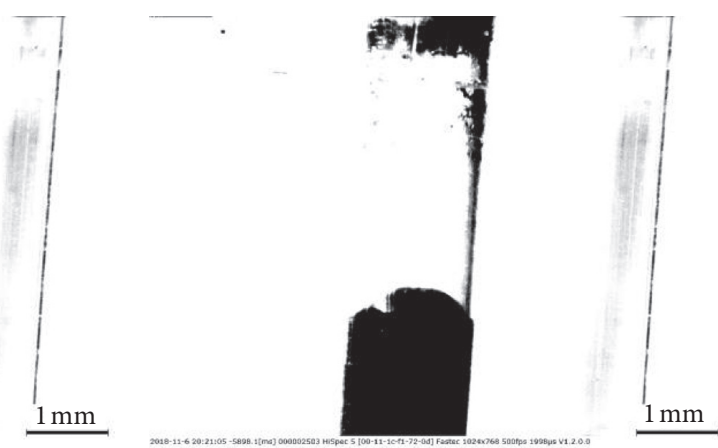

(d)
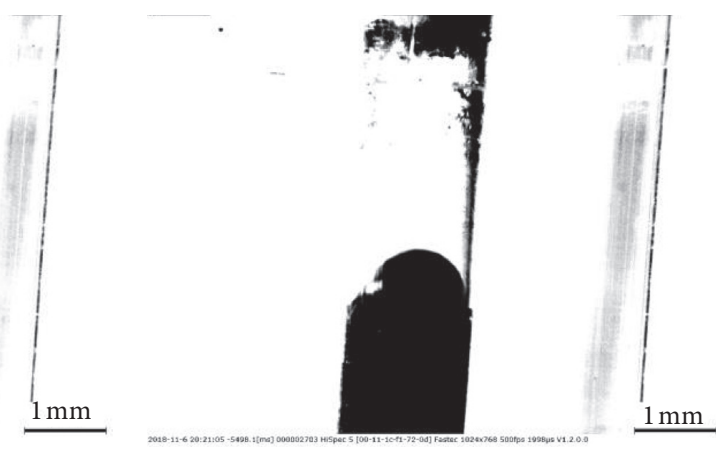

(f)

Figure 14: The test results of the start-up flow in the orifice: (a) $t=0 \mathrm{~s}$; (b) $t=0.6 \mathrm{~s}$; (c) $t=0.8 \mathrm{~s}$; (d) $t=1.0 \mathrm{~s}$; (e) $t=1.2 \mathrm{~s}$; (f) $t=1.4 \mathrm{~s}$.

TABLE 1: Parameters of the fractional K-BKZ numerical model.

\begin{tabular}{lcc}
\hline Parameter & Physical description & Numerical value \\
\hline$A$ & Acceleration & $1.0 \mathrm{~mm} / \mathrm{s}^{2}$ \\
$\mathrm{P}$ & Density & $1.1 \mathrm{~kg} / \mathrm{m}^{3}$ \\
$L$ & Orifice distance & $1.5 \mathrm{~mm}$ \\
$\Lambda$ & Relaxation time [39] & $1.94 \mathrm{~s}$ \\
$G(t)$ & Relaxation modulus [39] & $132.83 \mathrm{~Pa}$ \\
$A$ & Fractional order [39] & 0.35 \\
$\kappa$ & Quasiproperty & $1036.2 \mathrm{~Pa} \cdot \mathrm{s}^{\alpha}$ \\
$\mathrm{B}$ & Fractional order [39] & 0.8 \\
$\psi$ & Quasiproperty & $231.9 \mathrm{~Pa} \cdot \mathrm{s}^{\beta}$ \\
$A$ & A parameter related with the & 0.3 \\
\hline
\end{tabular}

The quasiproperties $\kappa$ and $\psi$ can be approximated by the relaxation modulus $G(t)$ and the fractional orders $\alpha$ and $\beta$, which can be written as [14] 


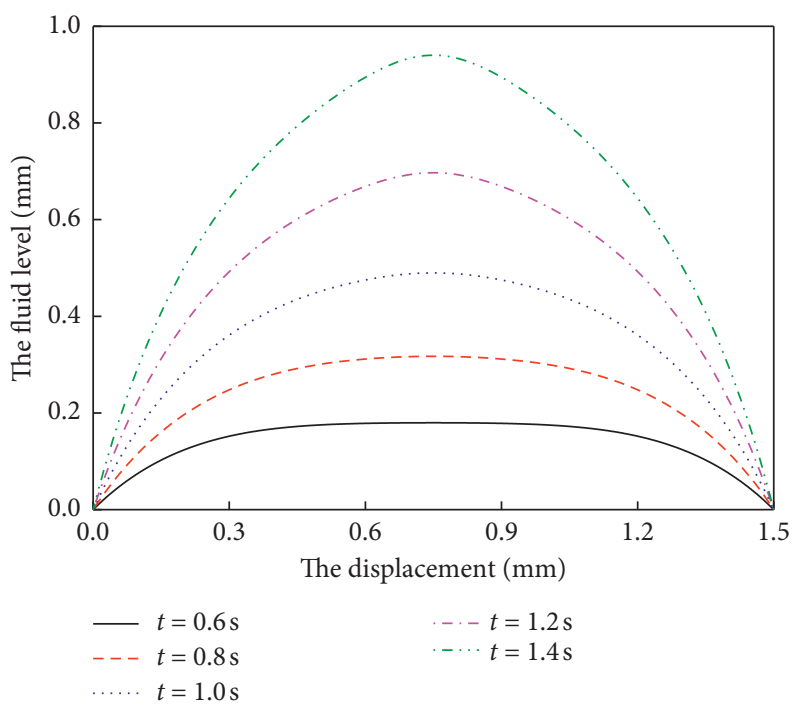

Figure 15: The fluid level distribution of the fractional K-BKZ numerical model in the orifice.

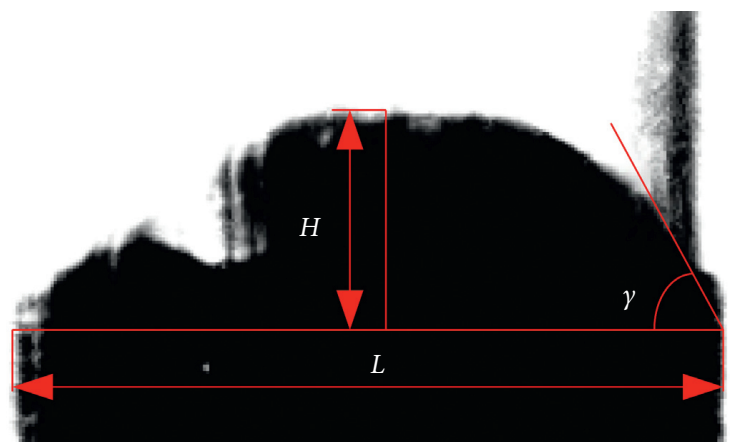

FIgURE 16: Viscoelastic fluid level measurement method ( $L$ is the orifice distance, $H$ is the maximum fluid level, and $\gamma$ is the contact angle).

TABle 2: Comparison of the maximum fluid level and the contact angle.

\begin{tabular}{|c|c|c|c|c|c|c|c|c|}
\hline $\begin{array}{l}\text { Test } \\
\text { results }\end{array}$ & $\begin{array}{c}H \\
(\mathrm{~mm})\end{array}$ & $\gamma\left({ }^{\circ}\right)$ & $\begin{array}{l}\text { The results of the fractional K-BKZ } \\
\text { numerical model }\end{array}$ & $\begin{array}{c}H \\
(\mathrm{~mm})\end{array}$ & $\Gamma\left(^{\circ}\right)$ & $\begin{array}{c}\text { The results of the Newtonian } \\
\text { numerical model }\end{array}$ & $\begin{array}{c}H \\
(\mathrm{~mm})\end{array}$ & $\gamma\left({ }^{\circ}\right)$ \\
\hline$t=0.6 \mathrm{~s}$ & 0.1889 & 44.4 & $t=0.6 \mathrm{~s}$ & 0.1800 & 45.6 & $t=0.6 \mathrm{~s}$ & 0.1800 & 29.5 \\
\hline$t=0.8 \mathrm{~s}$ & 0.3306 & 55.8 & $t=0.8 \mathrm{~s}$ & 0.3173 & 58.2 & $t=0.8 \mathrm{~s}$ & 0.3173 & 41.6 \\
\hline$t=0.1 \mathrm{~s}$ & 0.4665 & 62.3 & $t=0.1 \mathrm{~s}$ & 0.4889 & 65.2 & $t=0.1 \mathrm{~s}$ & 0.4889 & 52.8 \\
\hline$t=1.2 \mathrm{~s}$ & 0.6995 & 70.8 & $t=1.2 \mathrm{~s}$ & 0.6971 & 72.4 & $t=1.2 \mathrm{~s}$ & 0.6971 & 61.3 \\
\hline$t=1.4 \mathrm{~s}$ & 0.9075 & 74.4 & $t=1.4 \mathrm{~s}$ & 0.9402 & 76.7 & $t=1.4 \mathrm{~s}$ & 0.9402 & 67.7 \\
\hline
\end{tabular}

simulation results (as shown in Figure 15) and the test results (as shown in Figure 14) were then measured; the results have been shown in Table 2, and the trends have been shown in Figures 17 and 18.

As has been shown in Figure 17 and Table 2, the maximum liquid level of the two models was basically consistent with the tests, indicating that the models and the tests were well matched at the same time. It can be seen from Figure 18 and Table 2 that, as the time increased, the contact angles of the fractional K-BKZ numerical model were in good agreement with the tests, while those of the Newtonian fluid model were greatly different (the average error of the contact angle was $19.3 \%$ ), which meant that the fractional $\mathrm{K}-\mathrm{BKZ}$ numerical model showed strong viscoelasticity, while the Newtonian fluid model showed no viscoelasticity. The maximum error of the maximum fluid level was $4.79 \%$, and the maximum error of the contact angle was $4.65 \%$ (as shown in Figure 19). In summary, the fractional K-BKZ numerical model was able to accurately describe the viscoelastic characteristics of the viscoelastic material and accurately simulate the actual flow of the start-up flow in the shock absorber. 


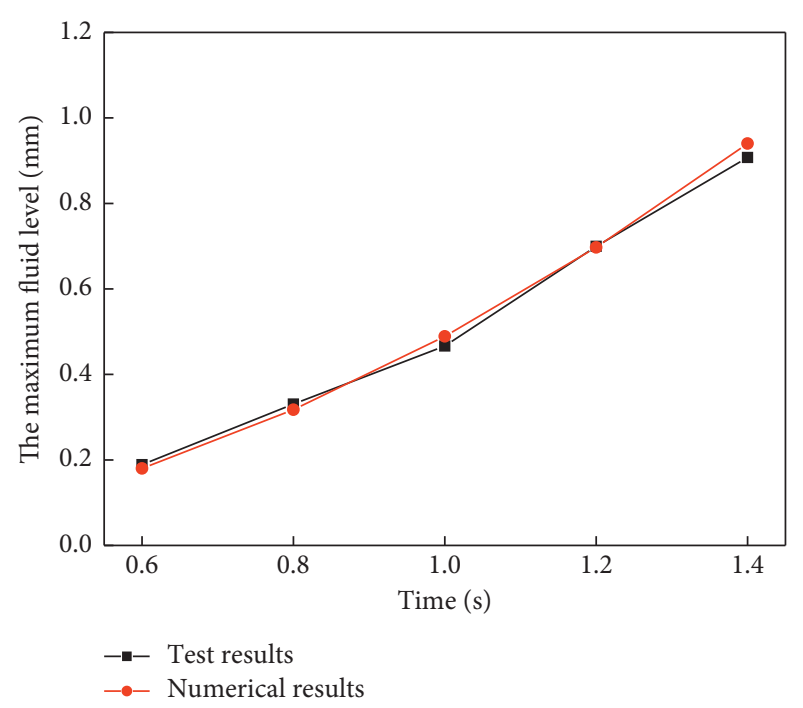

FIgURE 17: Comparison of the maximum fluid level.

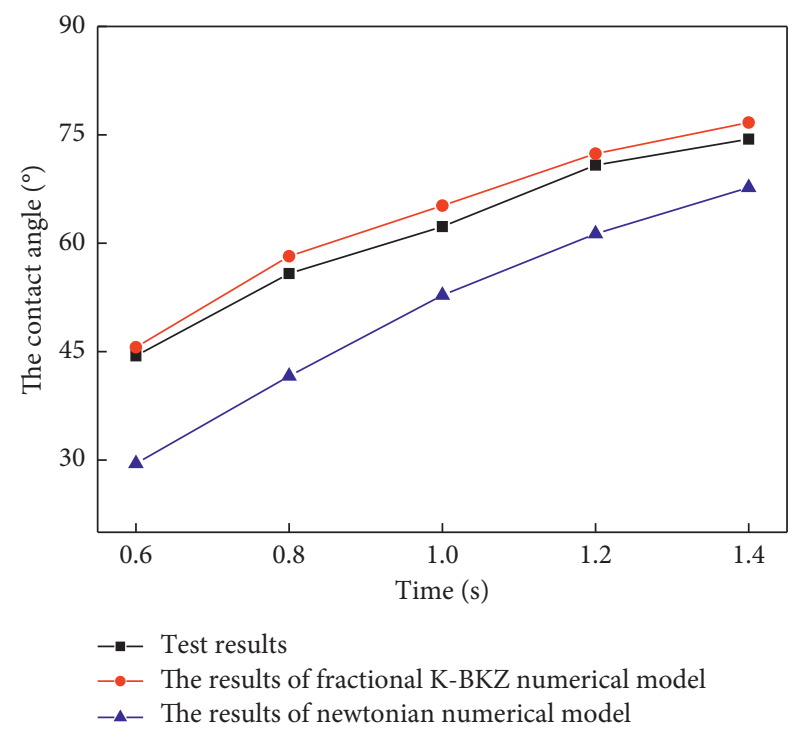

Figure 18: Comparison of the contact angle.

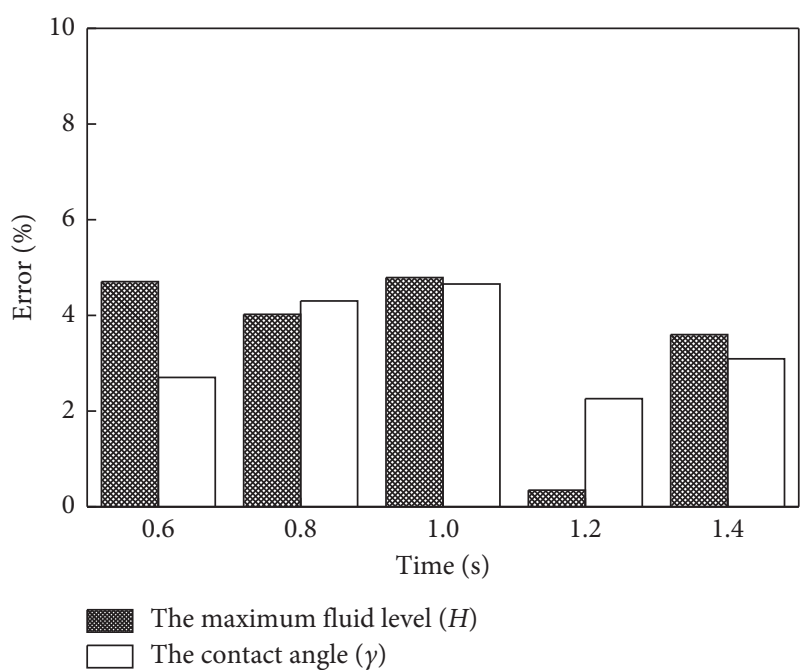

FIGURE 19: The error bars of the fractional K-BKZ numerical model.

\section{Conclusions}

In this paper, the fractional K-BKZ numerical model was proposed in order to study the start-up flows of the viscoelastic material in a shock absorber. According to the actual flow conditions of a viscoelastic material in the orifice and the gap of a shock absorber, the flow behavior was simplified to two forms: the accelerated flow of the viscoelastic fluid in a pipe and the flow of the viscoelastic fluid between two parallel plates with one plate accelerating and the other at rest.

The fractional K-BKZ numerical model has been established by adopting the framework of the classical $\mathrm{K}-\mathrm{BKZ}$ model and the relaxation modulus of the fractional Maxwell model with quasiproperties. According to the actual working environment, the initial conditions and the boundary conditions were set. Compared with the results given by the classical K-BKZ numerical model, the fractional Maxwell numerical model, and the Newtonian fluid model, the variations in the flow velocity and stress were analyzed. Finally, the test system for the start-up flow was designed to capture the flow process of the viscoelastic material in the orifice during the start-up flow. The maximum fluid level and the contact angle were defined and measured. The accuracy of the fractional $\mathrm{K}-\mathrm{BKZ}$ numerical model was verified by comparing the results from the model and the tests.

The numerical results and the test results proved that

(1) When the viscoelastic fluid had an accelerated flow in the pipe or between the two parallel plates, the flow velocity distribution simulated by the fractional K-BKZ numerical model always maintained good viscoelasticity. The classical K-BKZ numerical model and the fractional Maxwell numerical model lost the viscoelasticity and the accuracy of viscoelasticity model, respectively, when the time was long or the velocity was large. The Newtonian fluid model displayed no viscoelasticity.

(2) The fluid stresses increase for the fractional K-BKZ numerical model and the classical K-BKZ numerical model tended to be a steady state, which could effectively capture the shear-thinning phenomenon.

(3) Although the model accuracy did not change with the increase of the value of parameter $a$, the higher the values of parameter $\beta$ and $\vartheta$ were, the better the accuracy of the classical K-BKZ numerical model would be. With the increase of the parameters $\alpha, \beta$, and $\vartheta$, the velocity curve curvatures and the nonlinearity of the fractional K-BKZ numerical model gradually decreased, while the influence of the parameter $a$ was opposite to that of other parameters.

(4) The shape of the start-up flow of the viscoelastic material in the orifice developed from a flat shape to a parabolic shape. The comparison between the tests and the fractional K-BKZ numerical model proved that they had basically identical variation trends.

(5) The results of the maximum fluid level and the contact angle of the fractional K-BKZ numerical 
model were in good agreement with the test results. The maximum errors were $4.79 \%$ and $4.65 \%$, respectively, which proved the accuracy of the model. Thus, the fractional K-BKZ numerical model was able to accurately simulate the start-up flow of the viscoelastic material in a shock absorber, and the fractional $\mathrm{K}-\mathrm{BKZ}$ numerical model always maintained strong viscoelasticity.

\section{Data Availability}

The data used to support the findings of this study are available from the corresponding author upon request.

\section{Conflicts of Interest}

The authors declare they have no conflicts of interest.

\section{Acknowledgments}

Thanks to all of our colleagues for providing all types of help during the preparation of this manuscript.

\section{References}

[1] J. Jia, J. Du, Y. Wang, and H. Hua, "Design method for fluid viscous dampers," Archive of Applied Mechanics, vol. 78, no. 9, pp. 737-746, 2008.

[2] J. Jia, X. Shen, J. Du, Y. Wang, and H. Hua, "Design and mechanical characteristics analysis of a new viscous damper for piping system," Archive of Applied Mechanics, vol. 79, no. 3, pp. 279-286, 2009.

[3] S. F. Zhang, X. L. Liu, and Y. Liu, "High efficient viscoelastic damper for heavy trucks," Applied Mechanics and Materials, vol. 215-216, pp. 318-321, 2012.

[4] M. C. Constantinou and M. D. Symans, "Experimental study of seismic response of buildings with supplemental fluid dampers," The Structural Design of Tall Buildings, vol. 2, no. 2, pp. 93-132, 1993.

[5] R. Lewandowski and B. Chorążyczewski, "Identification of the parameters of the Kelvin-Voigt and the Maxwell fractional models, used to modeling of viscoelastic dampers," Computers \& Structures, vol. 88, no. 1-2, pp. 1-17, 2010.

[6] H. T. Qi and J. G. Liu, "Some duct flows of a fractional Maxwell fluid," The European Physical Journal Special Topics, vol. 193, no. 1, pp. 71-79, 2011.

[7] D. Yang and K.-Q. Zhu, "Start-up flow of a viscoelastic fluid in a pipe with a fractional Maxwell's model," Computers \& Mathematics with Applications, vol. 60, no. 8, pp. 2231-2238, 2010.

[8] B. H. Yan, L. Yu, and Y. H. Yang, "Study of oscillating flow in rolling motion with the fractional derivative Maxwell model," Progress in Nuclear Energy, vol. 53, no. 1, pp. 132-138, 2011.

[9] W. Tan and M. Xu, "Plane surface suddenly set in motion in a viscoelastic fluid with fractional Maxwell model," Acta Mechanica Sinica, vol. 18, no. 4, pp. 342-349, 2002.

[10] W. Tan, W. Pan, and M. Xu, "A note on unsteady flows of a viscoelastic fluid with the fractional Maxwell model between two parallel plates," International Journal of Non-linear Mechanics, vol. 38, no. 5, pp. 645-650, 2003.

[11] Y. Carrera, G. Avila-de La Rosa, E. J. Vernon-Carter, and J. Alvarez-Ramirez, "A fractional-order Maxwell model for
non-Newtonian fluids," Physica A: Statistical Mechanics and Its Applications, vol. 482, pp. 276-285, 2017.

[12] D. Yao, "A fractional dashpot for nonlinear viscoelastic fluids," Journal of Rheology, vol. 62, no. 2, pp. 619-629, 2018.

[13] C.-S. Sin, L. Zheng, J.-S. Sin, F. Liu, and L. Liu, "Unsteady flow of viscoelastic fluid with the fractional K-BKZ model between two parallel plates," Applied Mathematical Modelling, vol. 47, pp. 114-127, 2017.

[14] A. Jaishankar and G. H. McKinley, "A fractional K-BKZ constitutive formulation for describing the nonlinear rheology of multiscale complex fluids," Journal of Rheology, vol. 58, no. 6, pp. 1751-1788, 2014.

[15] E. Mitsoulis, "50 years of the K-BKZ constitutive relation for polymers," ISRN Polymer Science, vol. 2013, Article ID 952379, 22 pages, 2013.

[16] B. Bernstein, E. A. Kearsley, and L. J. Zapas, "A study of stress relaxation with finite strain," Transactions of the Society of Rheology, vol. 7, no. 1, pp. 391-410, 1963.

[17] L. L. Ferrás, N. J. Ford, M. L. Morgado et al., "A primer on experimental and computational rheology with fractional viscoelastic constitutive models," AIP Conference Proceedings, vol. 1843, no. 1, Article ID 020002, 2017.

[18] A. Jaishankar and G. H. McKinley, "Power-law rheology in the bulk and at the interface: quasi-properties and fractional constitutive equations," Proceedings of the Royal Society A: Mathematical, Physical and Engineering Sciences, vol. 469, no. 2149, Article ID 20120284, 2013.

[19] B. Bernstein, E. A. Kearsley, and L. J. Zapas, "Thermodynamics of perfect elastic fluids," Journal of Research of the National Bureau of Standards Section B Mathematics and Mathematical Physics, vol. 68B, no. 3, pp. 103-113, 1964.

[20] M. H. Wagner, "Analysis of time-dependent non-linear stress-growth data for shear and elongational flow of a lowdensity branched polyethylene melt," Rheologica Acta, vol. 15, no. 2, pp. 136-142, 1976.

[21] C. F. Wang and J. L. Kokini, "Simulation of the nonlinear rheological properties of gluten dough using the Wagner constitutive model," Journal of Rheology, vol. 39, no. 6, pp. 1465-1482, 1995.

[22] H. Yamaguchi, "Viscoelastic entrance flow of pipe with K-BKZ type model," Nihon Reoroji Gakkaishi (Journal of the Society of Rheology, Japan), vol. 22, no. 3, pp. 135-143, 1994.

[23] M. V. Bower, A. S. Wineman, and K. R. Rajagopal, "Flow of $\mathrm{K}-\mathrm{BKZ}$ fluids between parallel plates rotating about distinct axes: shear thinning and inertial effects," Journal of Nonnewtonian Fluid Mechanics, vol. 22, no. 3, pp. 289-307, 1987.

[24] M. Ebrahimi, V. K. Konaganti, and S. G. Hatzikiriakos, "Dynamic slip of polydisperse linear polymers using partitioned plate," Physics of Fluids, vol. 30, no. 3, Article ID 030601, 2018.

[25] M. F. Tomé, J. Bertoco, C. M. Oishi et al., "A finite difference technique for solving a time strain separable K-BKZ constitutive equation for two-dimensional moving free surface flows," Journal of Computational Physics, vol. 311, pp. 114141, 2016.

[26] R. B. Bird, R. C. Armstrong, and O. Hassager, "Single-integral constitutive equations," in Dynamics of Polymeric Liquids, pp. 425-476, John Wiley and Sons, New York, NY, USA, 1987.

[27] R. G. Larson, "Continuum theories," in Constitutive Equations for Polymer Melts and Solutions, pp. 75-87, Butterworths, Boston, MA, USA, 1988.

[28] V. H. Rolón-Garrido and M. H. Wagner, "The damping function in rheology," Rheologica Acta, vol. 48, no. 3, pp. 245-284, 2009. 
[29] M. H. Wagner and H. M. Laun, "Nonlinear shear creep and constrained elastic recovery of a LDPE melt," Rheologica Acta, vol. 17, no. 2, pp. 138-148, 1978.

[30] A. Jaishankar, "The linear and nonlinear rheology of multiscale complex fluids," Ph.D. dissertation, Massachusetts Institute of Technology, Cambridge, MA, USA, 2014.

[31] R. Gorenflo, A. Kilbas, F. Mainardi et al., "Introduction," in Mittag-Leffler Functions, Related Topics and Applications, vol. 2, pp. 1-7, Springer, Berlin, Germany, 2014.

[32] I. Podlubny, "Special functions of the fractional calculus," in Fractional Differential Equations: An Introduction to Fractional Derivatives, Fractional Differential Equations, to Methods of Their Solution and Some of Their Applications, pp. 16-37, Academic Press, San Diego, CA, USA, 1999.

[33] S. Hermann and G. Klaus, "Fundamentals of boundary-layer theory," in Boundary-Layer Theory, pp. 50-82, SpringerVerlag, Berlin, Germany, 2017.

[34] R. G. Larson, "Constitutive relationships for polymeric materials with power-law distributions of relaxation times," Rheologica Acta, vol. 24, no. 4, pp. 327-334, 1985.

[35] R. C. Koeller, "Applications of fractional calculus to the theory of viscoelasticity," Journal of Applied Mechanics, vol. 51, no. 2, pp. 299-307, 1984.

[36] A. J. Holder, N. Badiei, K. Hawkins, C. Wright, P. R. Williams, and D. J. Curtis, "Control of collagen gel mechanical properties through manipulation of gelation conditions near the sol-gel transition," Soft Matter, vol. 14, no. 4, pp. 574-580, 2018.

[37] Z. Q. Wang, B. Q. Mao, S. Feng et al., "Fractional Maxwell model of the start-up flow for a viscoelastic elastomer shock absorber," Acta Armamentarii, vol. 40, no. 10, pp. 1977-1986, 2019.

[38] R. Sharma and B. J. Cherayil, "Polymer melt dynamics: microscopic roots of fractional viscoelasticity," Physical Review E, vol. 81, no. 2, Article ID 021804, 2010.

[39] J. Jia, "Research on dissipating mechanism and design of the elastomer absorber," Ph.D. dissertation, Shanghai Jiaotong University, Shanghai, China, 2007.

[40] D. Y. Kwok and A. W. Neumann, "Contact angle measurement and contact angle interpretation," Advances in Colloid and Interface Science, vol. 81, no. 3, pp. 167-249, 1999. 